\title{
MicroED Structures from Micrometer Thick Protein Crystals
}

Michael W. Martynowycz ${ }^{1}$, Calina Glynn ${ }^{2}$, Jennifer Miao ${ }^{2}$, M. Jason de la

$$
\begin{gathered}
\text { Cruz }^{1} \text {, Johan Hattne }{ }^{1} \text {, Dan Shi }{ }^{1} \text {, Duilio Cascio }{ }^{3}, \\
\text { Jose Rodriguez }{ }^{2} \text {, and Tamir Gonen }{ }^{1,4^{*}}
\end{gathered}
$$

1 Janelia Research Campus, Howard Hughes Medical Institute, 19700 Helix Drive, Ashburn, Virginia 20147, USA

2 Department of Chemistry and Biochemistry; UCLA-DOE Institute; University of California, Los Angeles; Los Angeles, California 90095-1570, USA

${ }^{3}$ Department of Biological Chemistry, UCLA-DOE Institute; University of California, Los Angeles; Los Angeles, California 90095-1570, USA

${ }^{4}$ Departments of Biological Chemistry and Physiology, David Geffen Medical School, University of California, Los Angeles, California 90095-1570, USA.

* Correspondence should be addressed to T.G. (tgonen@ucla.edu) 


\title{
Summary
}

Atomic resolution protein structures can be determined by MicroED from crystals that surpass the theoretical maximum thickness limit by an order of magnitude.

\begin{abstract}
Theoretical calculations suggest that crystals exceeding $100 \mathrm{~nm}$ thickness are excluded by dynamical scattering from successful structure determination using microcrystal electron diffraction (MicroED). These calculations are at odds with experimental results where MicroED structures have been determined from significantly thicker crystals. Here we systematically evaluate the influence of thickness on the accuracy of MicroED intensities and the ability to determine structures from protein crystals one micrometer thick. To do so, we compare ab initio structures of a human prion protein segment determined from thin crystals to those determined from crystals up to one micrometer thick. We also compare molecular replacement solutions from crystals of varying thickness for a larger globular protein, proteinase K. Our results indicate that structures can be reliably determined from crystals at least an order of magnitude thicker than previously suggested by simulation, opening the possibility for an even broader range of MicroED experiments.
\end{abstract}




\section{Main Text}

\section{Introduction}

Electrons interact with matter more strongly than X-rays and offer a larger fraction of useful, elastic scattering events to inelastic scattering events (1). These properties are leveraged by the cryoEM method, MicroED, for structure determination at atomic resolution from nanoscale protein crystals (2). With this method, diffraction is measured from protein nanocrystals in a frozen-hydrated state (3) using a low dose electron beam (typically $\sim 0.01 \mathrm{e}^{-} \AA^{-2} \mathrm{~s}^{-1}$ ) (4-6). Crystals are continuously and unidirectionally rotated in the beam while diffraction images are collected as a movie on a fast detector (7). A number of previously unknown as well as known structures have been determined by this method to atomic resolution (8).

The strong interaction between electrons and materials also allows for multiple scattering events to take place before electrons exit the specimen (9). According to dynamical scattering theory (10), multiple scattering events produce inaccuracies in the recorded reflections, potentially preventing the solution of structures. Simulations suggest that with crystals thicker than $50-100 \mathrm{~nm}(11,12)$ dynamical scattering can be severe, resulting in nearly random intensities, where the relationship between the intensity and structure factor no longer holds true (10). However, these simulations assume diffraction is recorded from a perfect and stationary crystal - real macromolecular crystals are not perfect. In fact, precession electron diffraction can avoid many of the artifacts associated with dynamical scattering from near perfect crystals of inorganic material by pivoting of the electron beam around the crystal (13). 
Similarly, by employing continuous rotation MicroED (7), useful diffraction is routinely collected from protein crystals hundreds of nanometers thick (14). Even $1.5 \mu \mathrm{m}$-thick crystals of lysozyme were shown to produce diffraction that when integrated produced reasonable statistics (2). Moreover, recent structures determined by ab initio methods to $1 \AA$ resolution further indicate that the diffraction intensities obtained by continuous rotation MicroED are accurate and maintain the relationship between amplitude and phase $(4,15)$.

Here we systematically investigate the relationship between crystal thickness, dynamical scattering and the quality of structure solutions obtained by MicroED. We determine $a b$ initio structures of a segment from the $\beta_{2}-\alpha_{2}$ loop of human prion protein in the amyloid state as well as the structure of proteinase $\mathrm{K}$ by molecular replacement from crystals up to a micrometer thick.

\section{Results}

A comparison of structures determined from thin and thick crystals of a segment of the $\beta 2-\alpha 2$ loop of human prion protein. As a model system for evaluating the effects of crystal thickness on diffraction intensities, we use a segment from the $\beta 2-\alpha 2$ loop of human prion protein ( $\mathrm{hPrP}$ ) that contains a glycine residue at its amino terminus (sequence GSNQNNF), hereafter referred to as $\mathrm{hPrP}-\beta 2 \alpha 2$, for MicroED structure analysis from crystals that vary in thickness. The crystals of this segment appear as micrometer-long needles and vary in thickness from several nanometers to over a micrometer. Seven data sets originating from thin crystals were collected and combined 
to yield a reference data set that was $80.3 \%$ complete in P1 with constants $\{\mathbf{a}, \mathbf{b}, \mathbf{c}\}(\AA)$ $=\{4.86,14.11,18.41\}$, and angles $\{\alpha, \beta, \gamma\}\left(^{\circ}\right)=\{90.00,93.71,101.21\}$. A second data set was constructed from 7 thick crystals between 500 nanometers and one micrometer thick, that were combined to yield a $75.6 \%$ complete data set with the same space group and unit cell dimensions as above. Thin and thick crystal structures were determined ab initio by direct methods using SHELXT (16) (See SI Methods) and refined to atomic resolution using BUSTER-TNT (17) showing clear atomic density (Figure 1). Refinement statistics for hPrP- $\beta 2 \alpha 2$ are presented in Table 1.

The structure of hPrP- $\beta 2 \alpha 2$ represents a prion protofilament with amyloid features. The protofilament is a class 2 steric zipper, composed of parallel, face-to-back beta sheets (18). One pair of sheets makes the protofilament, as observed with other amyloid structures $(15,18)$. At this resolution, the density shows hydrogen atoms as well as the presence of zinc and acetate ions that facilitate crystallographic contacts; both were present in the crystallization condition (Figure 1). Structures from both thick and thin crystals show clear densities for waters in the $2 F_{0}-F_{c}$ map and have multiple hydrogens appearing in the $F_{o}-F_{c}$ density at the $3 \sigma$ level (Figure 1). The appearance of resolvable hydrogens in electron diffraction was first reported for proteins by Rodriguez et al. (15) and later by Palatinus (19) for small molecules. Our ab initio solutions from thin and thick crystals are very similar, with a backbone RMSD of $0.07 \AA$ and an all-atom RMSD of $0.09 \AA$ (Figure 1). In summary, we found no significant differences between structures determined from thin and thick crystals of hPrP- $\beta 2 \alpha 2$. 


\section{A comparison of structures determined from thin and thick crystals of a globular} protein. We collected MicroED data from single $\sim 500 \mathrm{~nm}$ and $\sim 1 \mu \mathrm{m}$-thick crystals of proteinase K (SI Figure 2, SI Figure 3) and determined structures from each. Reflections were recorded to a resolution of $1.8 \AA$ in both cases yielding $97 \%$ completeness for the $500 \mathrm{~nm}$ crystal and $79.7 \%$ completeness for the $1 \mu \mathrm{m}$ thick crystal. Data were reduced in XDS (20) and phased by molecular replacement using the atomic coordinates of PDBID 5i9s as a search model (21). Structures were refined using phenix.refine (22) with a high-resolution cutoff of $2.0 \AA$ and $3.0 \AA$, respectively (Table 1; Figure 2). As the single, $\sim 1$ micrometer thick crystal structure had poor statistics, its data were merged with data gathered from three additional crystals of proteinase $\mathrm{K}$; each of these also $\sim 1 \mu \mathrm{m}$ thick (SI Figure 4, SI Figure 5). The structure from the four combined data sets was determined again using the same search model now to a resolution of $2.5 \AA$ with better overall statistics (Table 1, Figure 2). A comparison of the resulting structures with the molecular replacement search model indicate good agreement with lower than $0.25 \AA$ all atom RMSD. In summary, we found no significant differences between structures determined from thin and thick crystals of proteinase $\mathrm{K}$.

\section{Systematic study of the effects of crystal thickness on MicroED data quality.}

Diffraction was measured from 19 crystals of $\mathrm{hPrP}-\beta 2 \alpha 2$ with thicknesses ranging from $\sim 100 \mathrm{~nm}$ to $\sim 1100 \mathrm{~nm}$ over similarly-sized wedges of reciprocal space corresponding to a real-space angular range of approximately $-30^{\circ}$ to $+30^{\circ}$ (SI Table 1). Data for all comparisons was indexed and integrated using XDS (20). Estimation of crystal thickness is discussed at length in SI Materials. Briefly, average crystal thickness is 
estimated by measuring the projected area from images recorded at $0^{\circ}$ and $60^{\circ}$ tilt, and the aspect ratio fit to an idealized model. These geometrical estimates were corroborated by intensity ratios using camera counts as previously described $(14,23)$ and are in good agreement (Si Figure 6). Crystal images are presented in SI Document 2. To assess the quality of diffraction data, we compare $R$ values $\left(R_{\text {meas }}\right)$, the ratio of intensity to variance $(I / \sigma \mathrm{l})$, and the half-set correlation coefficient $\left(\mathrm{CC}_{1 / 2}\right)$. All measured data values and statistics are presented in SI Table 1. Measured R values for these 19 crystals show a mean value of $13.03 \%$ with a standard deviation of $2.4 \%$; average I/OI values average 4.1 with a standard deviation of 0.91 . The half-set correlation coefficient is on average $98.2 \%$ with a standard deviation of $1.4 \%$. The structure factor amplitudes for these 19 crystals were compared to the hPrP- $\beta 2 \alpha 2$ structure solution from thin crystals discussed above. The correlation coefficient between the solved model and the individual crystals is shown in Figure-2D as $\mathrm{CC}_{\text {model }}$ (Figure 3; SI Table 1).

Absorption by protein crystals. The data presented above indicates that dynamical scattering does not inhibit structure solution by MicroED even from micrometer thick crystals when data is collected by continuous rotation. However, we note that the achievable resolution was lower from thick crystals compared to thin crystals (Figure 1,2 and Table 1). To evaluate whether absorption from thick crystals is limiting the achievable resolution, we recorded electron energy loss spectroscopy (EELS) spectra from 11 crystals of hPrP- $\beta 2 \alpha 2$ and 10 crystals of proteinase $\mathrm{K}$ with thicknesses ranging from $\sim 100 \mathrm{~nm}$ to $\sim 1400 \mathrm{~nm}$ at $300 \mathrm{kV}$ (Figure 4). Control spectra were recorded from 
regions of empty carbon support or within grid holes (SI Document 3). Each image was aligned by principal component analysis and a line scan through the zero-loss peak was measured along the first principal component. Intensities for the zero-loss peaks show expected exponential decay (9). A significant energy loss was observed for the carbon support alone with transmitted beam intensity decreasing by more than $40 \%$ (SI Document 3). An exponential fit to the data (Figure 4) suggests that the attenuation length, or (1/e) loss of intensity, due to a carbon film would be $\sim 87 \mathrm{~nm}$ at $300 \mathrm{kV}$. However, the (1/e) loss from crystals of proteinase $\mathrm{K}$ and hPrP- $\beta 2 \alpha 2$ are $261 \mathrm{~nm}$ and $323 \mathrm{~nm}$, respectively, at $300 \mathrm{kV}$. Our estimates of the actual carbon thickness are about 45nm using intensity ratios (SI Figure 6), in good agreement with previous findings on these grids (24). Thus even after accounting for the carbon film support, our estimates are very close to the mean free path of water at $300 \mathrm{kV}$, found experimentally to be $336 \mathrm{~nm}(25,26)$. This data suggests that even a three-fold decrease in intensity due to absorption is insufficient to prohibit structure solutions by MicroED for micrometer thick macromolecular crystals at $300 \mathrm{kV}$; the achievable resolution drops quickly near or beyond this thickness limit.

\section{Discussion}

These data indicate that accurate data can be collected and structures can be reliably determined by MicroED from crystals an order of magnitude thicker than previously expected. Simulations have suggested that structure determination by MicroED would be inhibited by dynamical scattering events from crystals only $\sim 50-100 \mathrm{~nm}$ thick $(11,27)$. Here we show that protein structure solutions can be obtained from substantially thicker 
crystals than simulated limits suggest for both globular proteins and peptides using both molecular replacement and ab initio methods, respectively.

High quality structure solutions can be obtained for both $\mathrm{hPrP}-\beta 2 \alpha 2$ and proteinase $\mathrm{K}$ regardless of data originating from thin crystals or crystals nearly one micrometer thick. Clear atomic density in the $2 \mathrm{~F}_{\mathrm{o}}-\mathrm{F}_{\mathrm{c}}$ map of $\mathrm{hPrP}-\beta 2 \alpha 2$ reveals hydrogen atoms and the presence of zinc and acetate ligands as well as ordered water molecules. Multiple hydrogens are apparent in the $F_{o}-F_{c}$ density at the $3 \sigma$ level (Figure 1). Likewise, the density for proteinase $\mathrm{K}$ is of high quality with well-defined density in both the $\sigma$ weighted difference maps and the SA composite omit maps for data obtained from thin $(<200 \mathrm{~nm})$, intermediate $(\sim 500 \mathrm{~nm})$ and thick $(>1000 \mathrm{~nm})$ crystals. Thickness does not prevent data collection, integration, or structure determination from these crystals.

The initial structure solution for proteinase $\mathrm{K}$ determined by MicroED (5i9s) was refined to a resolution of $1.75 \AA$ from merged data of four thin crystals (20). Our present models from single crystals of $\sim 500 \mathrm{~nm}$ and $\sim 1000 \mathrm{~nm}$ thickness are determined to $2 \AA$ and $3 \AA$ resolution, respectively. We credit this difference in resolution to increased absorption as demonstrated by our EELS analyses from thicker crystals. Together these results indicate that the achievable resolution can be limited by thick specimens. However, merging data from multiple thick crystals yielded in improved statistics and therefore improved resolution, indicating that absorption phenomena can be overcome to some extent by increased redundancy in measurements. Thicker protein crystals are ultimately limited in resolution by absorption. 
Why the discrepancy between the experimental data presented here and the limits found in previous simulations? The inherent difficulty of performing simulations requires assumptions of experimental conditions that fail to capture the complexity of MicroED experiments. Specifically, simulations assume that crystals are perfect, all the electrons are scattered and data is recorded from stationary crystals from major zone axes (11, 28). These conditions are often encountered in crystals of small molecules such as inorganic compounds in material science and from two-dimensional (2D) protein crystals, but are rarely encountered in macromolecular crystallography using 3D protein crystals and MicroED protocols (29). Crystals of macromolecules are highly mosaic compared with those of simple organic compounds or inorganic crystals. Macromolecular crystals are imperfect and bent at the nanoscale. MicroED data from highly mosaic crystals benefits from their disorder in analogy to the pivoting of an electron beam in precession electron diffraction. Moreover, MicroED data is collected by continuous rotation so integration over the rocking curve further curbs dynamical effects. Our results confirm that dynamic scattering is not a major problem in solving protein crystal structures, and that dynamic scattering effects do not increase linearly with crystal thickness. However, the question of why significant artifacts are not observed from multiple scattering still remains, and requires further study.

The fact that multiple scattering artifacts do not limit structure determination in MicroED is especially surprising for crystals thicker than $\sim 300 \mathrm{~nm}$, where the crystalline thickness easily exceeds the mean free path of an electron at either 200 or 300kV (Figure 4) (25, 
$26,30)$. For these crystals, few electrons are transmitted through the crystal without any interaction with the specimen. At very high thicknesses the diffraction spots begin to widen due to energy loss. Larger spots make crystals with many atoms per unit cell difficult to measure and may give poor statistics as a large fraction of scattering will be reduced by absorption. Integrating large broadened spots from crystals with large unit cells may result in spot-overlap and other complications that may limit the achievable resolution; we have yet to encounter this problem. The largest protein determined to date by MicroED is catalase at $\sim 240 \mathrm{kDa}$ and even with such a large unit cell and peak broadening, spot overlap was not observed and did not hinder structure determination (14). While broadening of diffraction spots can be curbed using an energy filter, this comes at the cost of reducing the transmitted signal. Nonetheless, use of an energy filter could improve signal to noise in MicroED experiments and the influence of thick crystals with large unit cells on diffraction quality remains to be further investigated.

In determining protein structures from crystals up to 1 micrometer in thickness using both $a b$ initio phase retrieval and molecular replacement, we demonstrate that MicroED experiments are ultimately limited by absorption effects and crystal quality. At present, we estimate crystals $\sim 500 \mathrm{~nm}$ or thinner maximize data quality $(\sim 2 \mathrm{x}$ mean free path for $200 \mathrm{kv}$ ), but structures from thicker crystals are not necessarily precluded. In fact, our results from even thicker crystals provides confidence that observed diffraction can be free of multiple scattering artifacts in a typical continuous rotation MicroED experiment, even from micrometer thick protein crystals. 


\section{Concluding Remarks}

We demonstrate that MicroED yields accurate diffraction intensities, and that structures can be determined by molecular replacement or ab initio methods from protein crystals much thicker than previously suggested. Our data demonstrates that, as with X-ray diffraction, dynamical scattering is not a prohibitive source of error when MicroED data is collected on protein crystals by continuous rotation. Instead, absorption phenomena limit the achievable resolution in a way that can be curbed by merging multiple data sets. We suggest a stringent upper bound on crystal thickness of about one micrometer on MicroED experiments and a soft upper bound of about 500nm to ensure high-quality diffraction with the best possible resolution; these limits are not imposed by dynamical scattering, but instead by absorption. Our study expands the usefulness of MicroED as a general method for structure determination to atomic resolution from specimens up to a micrometer thick, opening new avenues of research that may broadly impact structural biology.

\section{Materials and Methods}

Protein preparation. hPrP- $\beta 2 \alpha 2$ with greater than $98 \%$ purity was purchased from Genscript, dissolved in water at $10-20 \mathrm{mg} / \mathrm{ml}$ and screened by the hanging drop method in a high throughput screen. Initial hits were optimized in 24-well hanging drop trays. The best crystals were observed in a condition containing 10\% (w/v) PEG-8000; $0.1 \mathrm{M}$ MES pH 6.0; $\mathrm{Zn}(\mathrm{OAc})_{2}$. This condition was used as the basis for a batch crystallization of the peptide at $10 \mathrm{mg} / \mathrm{ml}$ at a $1: 1$ ratio of peptide solution to mother liquor. In this condition, crystals grew as needle clusters that could be broken by force of pipetting and applied to grids for cryopreservation. Proteinase K (E. Album) was purchased from 
Sigma and used without further purification. Crystals were grown by adding $5 \mu \mathrm{L}$ of protein solution $(50 \mathrm{mg} / \mathrm{ml})$ to $5 \mu \mathrm{L}$ of precipitant solution $(1.5 \mathrm{M}$ ammonium sulfate, 0.1 $\mathrm{M}$ Tris $\mathrm{pH}$ 8.0) in a sitting drop vapor diffusion tray. Large proteinase crystals were collected from sitting drops and sonicated into smaller crystals as previously described $(4)$

MicroED data collection. MicroED data was collected for 19 crystals of the peptide hPrP- $\beta 2 \alpha 2$ (Gly-Ser-Asn-Gln-Asn-Asn-Phe) of varying thickness over the real space wedge corresponding to $-30^{\circ}$ to $+30^{\circ}$ under continuous rotation. All MicroED experiments were performed on an FEI Tecnai F20 microscope at an accelerating voltage of $200 \mathrm{kV}$, corresponding to a wavelength of $0.0251 \AA$. Data was collected using a constant rotation rate of $0.2^{\circ} \mathrm{s}^{-1}$ on a TVIPS TemCam-F416 CMOS detector in rollingshutter mode with $5 \mathrm{~s}$ exposures. Beam intensity for all hPrP- $\beta 2 \alpha 2$ crystals was constant with an average dose rate of $0.003 \mathrm{e}^{-} \AA^{-1} \sec ^{-1}$, or an overall exposure of $\sim 1 \mathrm{e}^{-}$ $\AA^{-2}$. Proteinase $K$ crystals were collected at a dose rate of $0.01 \mathrm{e}^{-} \AA^{-1} \sec ^{-1}$ with an overall total dose per crystal of less than $3 e^{-} \AA^{-2}$. hPrP- $\beta 2 \alpha 2$ samples maintained a camera length of $0.730 \mathrm{~m}$ equivalent to a sample-detector distance of $1.313 \mathrm{~m}$ in a corresponding lens less system. Proteinase $\mathrm{K}$ data were collected at a camera length of $1.2 \mathrm{~m}$, corresponding to a detector distance of $2.200 \mathrm{~m}$. Diffraction data were collected through a circular selected area aperture of $1 \mu \mathrm{m}^{2}$ in projection to reduce background noise. All TEM measurements were done at liquid nitrogen temperatures ( $77 \mathrm{~K})$. 
Determination of Crystal Thickness. Crystal thickness was assessed in two ways: geometrical measurement and by using counts on the camera. First, Average crystal thickness was initially estimated geometrically by examining the projected area of each crystal from images taken at $0^{\circ}, 15^{\circ}, 30^{\circ}, 45^{\circ}$, and $60^{\circ}$ using the pixel length calibration from the known grid hole size of either 1 or $2 \mu \mathrm{m}$ in ImageJ (NIH). Crystals of hPrP$\beta 2 \alpha 2$ were all rod-shaped and assumed to be ellipsoidal rods. Tilt series assessed the aspect ratio of the major (a) and minor (b) axis at each angle, with a known length of $2 \mathbf{a}$ being the rod width measured at $0^{\circ}$. The average thickness of a crystal is then $\pi / 4=$ $\sim 0.785 \%$ of the maximum thickness, $2 \mathbf{b}$, perpendicular to the film. Proteinase $\mathrm{K}$ crystals were assumed to be cuboids with edge lengths $\mathbf{w}, \mathbf{I}, \mathbf{h}$. Edge lengths of $\mathbf{w}$ and I were found from the $0^{\circ}$ images and the $\mathbf{h}$ edge was estimated by the change in projected area of the crystal over a fixed length along the $\mathbf{x}$ and $\mathbf{y}$ axis. The average thickness of these crystals was assumed to be equal to the estimation of the $\mathbf{h}$ edge. Measurement accuracy was between 5 and $10 \mathrm{~nm}$, the average variance for each crystal was 20\%, and the standard deviation between intensity and geometry estimations was $\sim 100 \mathrm{~nm}$. Second, The geometrical approximation of crystal thickness above was validated by using intensity ratios as previously described $(2,14,26)$. Each crystal from the hPrP$\beta 2 \alpha 2$ set in Figure-3/SI Document 2 at $0^{\circ}$ were identified at $0^{\circ}$. Each crystal had an area selected from the portion of the crystal collected upon where the median intensity transmitted was calculated. The same area was then used to calculate the median intensity for the nearest clean carbon area and nearest empty grid hole for comparison. The crystal thickness was estimated by first estimating the carbon thickness using the formula given in Feja \& Aebi (26) assuming all missing intensity was lost due to energy 
loss signals and the incident maximum intensity was that of the beam intensity in a grid hole. The mean free path of the amorphous carbon was calculated using the average energy loss of carbon being $14.1 \mathrm{eV}$ derived from the formula given in (26), with $\mathrm{Z}=6$, corresponding to a mean free path of $125 \mathrm{~nm}$ at $200 \mathrm{kV}$. This gave a consistent value of the carbon thickness being approximately $45 \mathrm{~nm}$ with a standard deviation of $7 \mathrm{~nm}$. The crystal thickness is then calculated by subtracting the contribution of the carbon using the same equation with a mean free path of $242 \mathrm{~nm}$, calculated by scaling the value of $203 \mathrm{~nm}$ mean free path of vitreous ice given by Grimm et al. at $120 \mathrm{kV}(25,30)$. Our two measures were found to have a standard deviation of approximately $100 \mathrm{~nm}$. The correlation coefficient between the two measures was found to be $89 \%$. This suggests our geometrical measurements are accurate within $25 \%$ of our listed values. The correlation plot and deviations from these intensity measures are given in SI Figure 6.

Absorption Experiments. Energy spectra were collected on a JEOL JEM-3200FSC microscope at $300 \mathrm{kV}$ with a $500 \mathrm{~nm}^{2}$ aperture in projection and total dose of $0.1 \mathrm{e}^{-} \AA^{-2}$. Spectra were collected on a TVIPS TemCam-F416 CMOS detector at full resolution in normal (integration) mode and saved as 16-bit signed integer TIFF files. Spectra were scaled in ImageJ to the known $100 \mathrm{eV} \mathrm{mm}^{-1}$ energy filter spacing and $15.6 \mu \mathrm{m}$ pixel size. Individual images were loaded into Mathematica and aligned with their principal component along the horizontal axis - typically resulting in a $6.7^{\circ}$ clockwise rotation. Line scans of width 1 pixel were selected through the zero-loss peak along the principal loss axis, and shifted such that their peak intensities were located at an energy loss of 0 eV. Thickness for crystals used in spectra were determined as described above. Zero- 
loss peaks were fit to a general Gaussian model of $I=a^{*} e^{-b(x-c) 2}$ to determine peak maxima and integrated peak intensity. Data points were weighted by the square root of their intensities for the fitting to assure proper solutions. Zero-loss peak intensity was fit to a general exponential decay model with the intercept fixed to the normalized value of the vacuum peak as in Figure-4. Exponential fits to the experimental data for the carbon film, proteinase $\mathrm{K}$, and $\mathrm{hPrP}-\beta 2 \alpha 2$ protein crystals resulted in $\mathrm{R}^{2}$ residuals of 1.0 , 0.85 , and 0.88 , respectively.

MicroED Data Processing. Diffraction movies were converted to the SMV file format using TVIPS tools (31) and checked for pixel truncation as previously described (21). Indexing and integration were performed in XDS $(20,32)$. Integrated diffraction intensities were sorted and merged in XSCALE. Merged intensities were converted to amplitudes and the files formatted to SHELX format in XDSCONV. Thick and thin hPrP$\beta 2 \alpha 2$ were solved using SHELXT, placing all of the atoms in the unit cell correctly (33). SHELXT supported the hypothesis of P1 crystallographic symmetry (16). Intensities for proteinase K were input directly from XSCALE into Phaser for molecular replacement (34). Molecular replacement was successful with a LLG > 1000 and a TFZ > 20 using the model 5i9s. Refinement was carried out using phenix.refine (22). Individual models were adjusted manually in Coot (35) by visual inspection of the atomic model against the $F_{o}-F_{c}$ and $2 F_{o}-F_{c}$ maps. All-atom composite omit maps with simulated annealing were calculated in Phenix (22). 
Models and Figures. Figures were generated in either PyMol or VMD (36, 37). Plots and fits to data such as EELS spectra were created in Mathematica using nonlinear model fits to the data. Backbone RMSDs were calculated in VMD. All atom RMSD values were generated by the align command in PyMol.

\section{Acknowledgements}

We thank David Eisenberg (HHMI, UCLA), Robert Glaeser (UCB), and Michael Sawaya (UCLA) for helpful discussions and/or critical reading of this manuscript. Jose Rodriguez is supported as a Searle Scholar and a Beckman Young Investigator. The Gonen Laboratory is supported by the Howard Hughes Medical Institute. This work was also supported by the Janelia Research Visitor Program.

\section{References}

1. Henderson R (2009) The potential and limitations of neutrons, electrons and Xrays for atomic resolution microscopy of unstained biological molecules. $Q \operatorname{Rev}$ Biophys 28(2):171.

2. Shi D, Nannenga BL, ladanza MG, Gonen T (2013) Three-dimensional electron crystallography of protein microcrystals. Elife 2:e01345.

3. Taylor KA, Glaeser RM (1974) Electron Diffraction of Frozen, Hydrated Protein Crystals. Science (80- ) 186(4168):1036-1037.

4. de la Cruz MJ, et al. (2017) Atomic-resolution structures from fragmented protein crystals with the cryoEM method MicroED. Nat Methods (February):1-6.

5. Nannenga BL, Gonen T (2016) MicroED opens a new era for biological structure determination. Curr Opin Struct Biol 40:128-135. 
6. Liu S, et al. (2016) Atomic resolution structure determination by the cryo-EM method MicroED. Protein Sci 26:8-15.

7. Nannenga BL, Shi D, Leslie AGW, Gonen T (2014) High-resolution structure determination by continuous-rotation data collection in MicroED. Nat Methods 11(9):927-30.

8. Nannenga BL, Gonen T (2016) MicroED opens a new era for biological structure determination. Curr Opin Struct Biol 40:128-135.

9. Kirkland EJ (2010) Advanced computing in electron microscopy doi:10.1007/9781-4419-6533-2.

10. Cowley JM, Moodie AF (1959) The scattering of electrons by atoms and crystals. II. The effects of finite source size. Acta Crystallogr 12(5):353-359.

11. Subramanian G, Basu S, Liu H, Zuo JM, Spence JCH (2015) Solving protein nanocrystals by cryo-EM diffraction: Multiple scattering artifacts. Ultramicroscopy 148:87-93.

12. Glaeser RM, Downing KH (1993) High-resolution electron crystallography of protein molecules. Ultramicroscopy 52(3-4):478-486.

13. White TA, Eggeman AS, Midgley PA (2010) Is precession electron diffraction kinematical? Part I: "Phase-scrambling" multislice simulations. Ultramicroscopy 110(7):763-770.

14. Nannenga BL, Shi D, Hattne J, Reyes FE, Gonen T (2014) Structure of catalase determined by MicroED. Elife 3:e03600.

15. Rodriguez JA, et al. (2015) Structure of the toxic core of a-synuclein from invisible crystals. Nature advance on(7570):486-490. 
16. Sheldrick GM (2015) SHELXT - Integrated space-group and crystal-structure determination. Acta Crystallogr Sect A Found Crystallogr 71(1):3-8.

17. Blanc E, et al. (2004) Refinement of severely incomplete structures with maximum likelihood in BUSTER-TNT. Acta Crystallogr Sect D Biol Crystallogr 60(12 I):2210-2221.

18. Sawaya MR, et al. (2007) Atomic structures of amyloid cross- $\beta$ spines reveal varied steric zippers. Nature 447(7143):453-457.

19. Palatinus L, et al. (2017) Hydrogen positions in single nanocrystals revealed by electron diffraction. Science (80- ) 355(6321). doi:10.1126/science.aak9652.

20. Kabsch W (2010) Xds. Acta Crystallogr Sect D Biol Crystallogr 66(2):125-132.

21. Hattne J, Shi D, De La Cruz MJ, Reyes FE, Gonen T (2016) Modeling truncated pixel values of faint reflections in MicroED images. J Appl Crystallogr 49:1029_ 1034.

22. Afonine P V., et al. (2012) Towards automated crystallographic structure refinement with phenix.refine. Acta Crystallogr Sect D Biol Crystallogr 68(4):352367.

23. Malis T, Cheng SC, Egerton RF (1988) EELS log-ratio technique for specimenthickness measurement in the TEM. J Electron Microsc Tech 8(2):193-200.

24. Cho H-J, et al. (2013) Measurement of ice thickness on vitreous ice embedded cryo-EM grids: investigation of optimizing condition for visualizing macromolecules. J Anal Sci Technol 4(1):7.

25. Grimm R, Typke D, Bärmann M, Baumeister W (1996) Determination of the inelastic mean free path in ice by examination of tilted vesicles and automated 
most probable loss imaging. Ultramicroscopy 63(3-4):169-179.

26. Feja B, Aebi U (1999) Determination of the inelastic mean free path of electrons in vitrified ice layers for on-line thickness measurements by zero-loss imaging. $J$ Microsc 193(1):15-19.

27. Grigorieff N, Henderson R (1996) Comparison of calculated and observed dynamical diffraction from purple membrane: Implications. Ultramicroscopy 65(12):101-107.

28. Jiang L, Georgieva D, Nederlof I, Liu Z, Abrahams JP (2011) Image Processing and Lattice Determination for Three-Dimensional Nanocrystals. Microsc Microanal 17(6):879-885.

29. Shi D, et al. (2016) The collection of MicroED data for macromolecular crystallography. Nat Protoc 11(5):895-904.

30. Russo CJ, Passmore LA (2016) Progress towards an optimal specimen support for electron cryomicroscopy. Curr Opin Struct Biol 37:81-89.

31. Hattne J, et al. (2015) MicroED data collection and processing. Acta Crystallogr Sect A Found Adv 71:353-360.

32. Kabsch W (2010) Integration, scaling, space-group assignment and postrefinement. Acta Crystallogr Sect D Biol Crystallogr 66(2):133-144.

33. Sheldrick GM (2008) A short history of SHELX. Acta Crystallogr Sect A Found Crystallogr 64(1):112-122.

34. McCoy AJ, et al. (2007) Phaser crystallographic software. J Appl Crystallogr 40(4):658-674.

35. Emsley P, Cowtan K (2004) Coot: Model-building tools for molecular graphics. 
Acta Crystallogr Sect D Biol Crystallogr 60(12 I):2126-2132.

36. DeLano W (2002) PyMOL. DeLano Sci San Carlos, CA (June 1972):1-15.

37. Humphrey W, Dalke A, Schulten K (1996) VMD: Visual molecular dynamics. J Mol Graph 14(1):33-38.

\section{Table and Figure Legends}

Table-1: Data Collection and Refinement Statistics.

Figure-1: Structural comparison of hPrP- $\beta 2 \alpha 2$ from thin and thick crystals by direct methods (A) Structural model for $h P r P-\beta 2 \alpha 2$ solved from typically $<200 \mathrm{~nm}$ crystals (B) from a set of crystals with average thicknesses $>1 \mu \mathrm{m} .2 \mathrm{mF}_{\mathrm{o}}-\mathrm{DF} \mathrm{F}_{\mathrm{c}}$ and $\mathrm{mFo}-$ DFc density maps are contoured at $1.5 \sigma$ and $3 \sigma$ levels, respectively. Positive hydrogen densities for both solutions are visible in $\mathbf{A}$ and $\mathbf{B}$ in green and are designed by orange arrows. (C) Ab initio solutions from both normal (yellow) and $>1 \mu \mathrm{m}$ (blue) peptide crystals. (D) Final structural model of hPrP- $\beta 2 \alpha 2$.

Figure-2: Structural comparison of globular proteins determined from thin, intermediate and thick crystals. Proteinase $\mathrm{K}$ sidechain density and corresponding structure solution from (A) thin $\sim 200 \mathrm{~nm}$ crystals (PDBID 5I9S)(20), (B) a single $600 \mathrm{~nm}$ thick crystal, (C) a single $1000 \mathrm{~nm}$-thick crystal, and (D) four crystals thicker than 1000 $\mathrm{nm}$. All $2 \mathrm{~F}_{\mathrm{o}}-\mathrm{F}_{\mathrm{c}}$ density maps are contoured at the $1.5 \sigma$ level for residues $226-240$ shown as a grey mesh. (E) All structure solutions of proteinase $\mathrm{K}$ overlaid for comparison. Overlaid colors correspond to individual colored structures. 
Figure-3: Measurements from hPrP- $\beta 2 \alpha 2$ crystals of variable thickness. (A) Mean I/ ol values, (B) Measured, expected $R$ values and their difference, $R_{\text {diff, }}(\mathbf{C})$ internal half set correlation coefficient, and (D) the correlation of the measured structure factors to the solved thin crystal model, or $\mathrm{CC}_{\text {solved. }}$ Mean values are depicted by solid lines. The dotted black line corresponds to the maximum reported limit from simulations (10).

Figure-4: Observed transmission from crystals of various thicknesses. Peak intensities of the electron energy loss spectroscopy (EELS) spectra obtained from both hPrP- $\beta 2 \alpha 2$ (blue) and proteinase $\mathrm{K}$ (orange) crystals. Black dots correspond to the recorded vacuum intensity and the carbon film. Exponential decay models are presented as dashed lines coordinated to the sample color. The thickness limit for MicroED experiments suggested by simulatins is given as a dashed horizontal line (10).

SI-Table-1: MicroED diffraction data from hPrP- $\beta 2 \alpha 2$ crystals of various thicknesses.

SI-Figure-1: (A) Thick crystals used to solve the thick hPrP- $\beta 2 \alpha 2$ structure. (B) Structure solution of hPrP- $\beta 2 \alpha 2$ solved from 7 crystals with average thickness $>1 \mu \mathrm{m}$. Colors show individually measured angular ranges between -65 and -63 degrees with corresponding crystals having matching border colors. Grey regions correspond to the angular wedge not measured and (orange) showing the region available for measurement. Scale bars correspond to $2 \mu \mathrm{m}$. 
SI-Figure-2: (A) Single $500 \mathrm{~nm}$ crystal used to solve the proteinase $\mathrm{K}$ structure at marked angles and locations. (B) Corresponding structure solution model. Measurable data after inclusion of symmetry (orange) and recorded angular range (blue) from the crystal. Grey angular regions depict ranges not measured. Scale bars correspond to 2 $\mu \mathrm{m}$.

SI-Figure-3: (A) Single $1 \mu \mathrm{m}$ crystal used to solve the proteinase $\mathrm{K}$ structure at marked angles and locations. (B) Corresponding structure solution model. Measurable data after inclusion of symmetry (orange) and recorded angular range (blue) from the crystal. Grey angular regions depict ranges not measured. Scale bars correspond to $2 \mu \mathrm{m}$.

SI-Figure-4: (A) Four $>1 \mu \mathrm{m}$ crystals used to solve the proteinase $\mathrm{K}$ structure at marked angles and locations. (B) Corresponding structure solution model. Measurable data after inclusion of symmetry (orange) and recorded angular range (blue) from the crystal. Grey angular regions depict ranges not measured. Scale bars correspond to $2 \mu \mathrm{m}$.

SI-Figure-5: $2 \mathrm{mF}_{\mathrm{o}}-\mathrm{DF} \mathrm{F}_{\mathrm{c}}$ and all-atom $\mathrm{SA}$ composite omit potential maps with corresponding structural model for proteinase $\mathrm{K}$ from (A) four thin crystals, (B) a single $600 \mathrm{~nm},(\mathbf{C})$ a single $1 \mu \mathrm{m}$, and (D) four merged crystals $>1 \mu \mathrm{m}$ thick. $2 \mathrm{~F}_{\mathrm{o}}-\mathrm{F}_{\mathrm{c}}$ potential maps are contoured at the $1.5 \sigma$ level with a $2 \AA$ curve.

SI-Figure-6: Crystal thickness estimation. (A) Comparison between the geometrically measured thickness (orange) and the thickness estimate from the Beer-Lambert Law 
(Grey), and the calculated carbon thickness from the Beer-Lambert calculation (Blue).

(B) Correlation between the two types of measures of crystal thickness.

SI-Document-2: Peptide tilt angle images (diffraction data crystals) - PDF

SI-Document-3: EELS data (crystals, spectra, and fits) - PDF 


\begin{tabular}{|c|c|c|c|c|c|c|}
\hline & hPrP- $\beta 2 \alpha 2$ & Thick hPrP- $\beta 2 \alpha 2$ & Proteinase $\mathrm{K}$ & $\begin{array}{c}\text { Proteinase K } \\
\text { (Intermediate Thickness) }\end{array}$ & $\begin{array}{c}\text { Proteinase } K \\
(>1 \mu \mathrm{m}, \text { single crystal) }\end{array}$ & $\begin{array}{c}\text { Proteinase } K \\
\text { (>1 } \mathrm{m} \text {, multiple crystals) }\end{array}$ \\
\hline Wavelength $(\AA)$ & 0.0251 & 0.0251 & 0.0251 & 0.0251 & 0.0251 & 0.0251 \\
\hline Resolution range $(\AA)$ & $7.608-1.001(1.037-1.001)$ & $14.03-1.011(1.047-1.011)$ & $19.62-1.75(1.813-1.75)$ & $19.39-2.0(2.072-2.0)$ & $22.16-3.001(3.108-3.001)$ & $22.61-2.5(2.589-2.5)$ \\
\hline Space group (\#) & P $1(1)$ & P $1(1)$ & P 43212 (96) & P 43212 (96) & P $43212(96)$ & P 43212 (96) \\
\hline $\begin{array}{c}\text { Unit cell } \\
\left.\text { a, b, c ( }()^{2}\right) \\
\alpha, \beta, \gamma\left({ }^{\circ}\right)\end{array}$ & $\begin{array}{l}4.8614 .1118 .41 \\
9093.71101 .21\end{array}$ & $\begin{array}{c}14.344 .8917 .8 \\
94.9691 .02101 .82\end{array}$ & $\begin{array}{c}67.3267 .32101 .01 \\
909090\end{array}$ & $\begin{array}{c}67.2167 .21100 .59 \\
909090\end{array}$ & $\begin{array}{c}68.0968 .09102 .13 \\
909090\end{array}$ & $\begin{array}{c}67.567 .5102 .41 \\
909090\end{array}$ \\
\hline Total reflections & $12907(524)$ & $8654(307)$ & $104808(5429)$ & $57404(4205)$ & $20317(1505)$ & $78690(5656)$ \\
\hline Unique reflections & $2072(171)$ & $1869(141)$ & $22698(2256)$ & $15756(1543)$ & $4135(294)$ & $8627(828)$ \\
\hline Multiplicity & $6.2(4.2)$ & $4.6(3.2)$ & $4.6(4.4)$ & $3.6(3.6)$ & $4.9(5.1)$ & $9.1(9.2)$ \\
\hline Completeness (\%) & $80.29(69.08)$ & $75.90(60.00)$ & $94.02(94.14)$ & $97.18(97.60)$ & $79.70(81.82)$ & $99.41(99.64)$ \\
\hline Mean I/sigma(I) & $5.05(2.25)$ & $4.64(1.65)$ & $3.1(1.0)$ & $3.0(0.87)$ & $2.20(0.92)$ & $4.83(2.39)$ \\
\hline Wilson B-factor & 4.38 & 6.55 & 11.77 & 24.23 & 39.66 & 17.86 \\
\hline R-merge & $0.238(0.43)$ & $0.233(0.474)$ & $0.458(1.989)$ & $0.294(1.28)$ & $0.551(1.47)$ & $0.414(0.960)$ \\
\hline R-meas & $0.257(0.485)$ & $0.258(0.552)$ & $0.516(2.258)$ & $0.347(1.51)$ & $0.617(1.63)$ & $0.44(0.97)$ \\
\hline $\mathrm{CC}_{1 / 2}$ & $0.98(0.86)$ & $0.979(0.489)$ & $0.929(0.112)$ & $0.972(0.289)$ & $0.883(0.316)$ & $0.939(0.484)$ \\
\hline Reflections used in refinement & $2072(172)$ & $1869(141)$ & $22670(2235)$ & 15756 (1544) & $4135(414)$ & $8627(828)$ \\
\hline Reflections used for R-free & $207(17)$ & $180(12)$ & $1476(146)$ & $788(77)$ & $207(20)$ & $431(41)$ \\
\hline R-work & $0.1984(0.2067)$ & $0.1925(0.2102)$ & $0.217(0.348)$ & $0.2218(0.3112)$ & $0.1987(0.3059)$ & $0.2085(0.2858)$ \\
\hline R-free & $0.2382(0.222)$ & $0.2523(0.2920)$ & $0.266(0.418)$ & $0.2508(0.3390)$ & $0.2497(0.4124)$ & $0.2612(0.4195)$ \\
\hline Number of non-hydrogen atoms & 66 & 63 & 2170 & 2130 & 2037 & 2090 \\
\hline macromolecules & 55 & 55 & 2027 & 2027 & 2027 & 2027 \\
\hline ligands & 5 & 5 & 10 & 10 & 10 & 10 \\
\hline solvent & 6 & 3 & 133 & 93 & 0 & 53 \\
\hline Protein residues & 7 & 7 & 281 & 281 & 281 & 281 \\
\hline RMS (bonds) & 0.014 & 0.013 & 0.003 & 0.004 & 0.005 & 0.003 \\
\hline RMS (angles) & 1.82 & 1.69 & 0.57 & 0.74 & 0.92 & 0.7 \\
\hline Ramachandran favored (\%) & 100 & 100 & 97.11 & 97.11 & 97.11 & 97.11 \\
\hline Ramachandran allowed (\%) & 0 & 0 & 2.53 & 2.53 & 2.53 & 2.53 \\
\hline Ramachandran outliers (\%) & 0 & 0 & 0.36 & 0.36 & 0.36 & 0.36 \\
\hline Rotamer outliers (\%) & 0 & 0 & 0 & 0 & 0.94 & 0 \\
\hline Clashscore & 0 & 0 & 4.54 & 4.81 & 3.29 & 2.28 \\
\hline Average B-factor & 5.85 & 6.78 & 11.49 & 23.19 & 25.12 & 12.61 \\
\hline macromolecules & 4.76 & 6.05 & 11.19 & 23.28 & 25.15 & 12.75 \\
\hline ligands & 7.48 & 9.69 & 29.54 & 27.28 & 19.83 & 16.75 \\
\hline solvent & 14.52 & 15.18 & 14.63 & 20.75 & 0 & 6.39 \\
\hline
\end{tabular}


A. hPrP- $\beta 2 \alpha 2$ structure

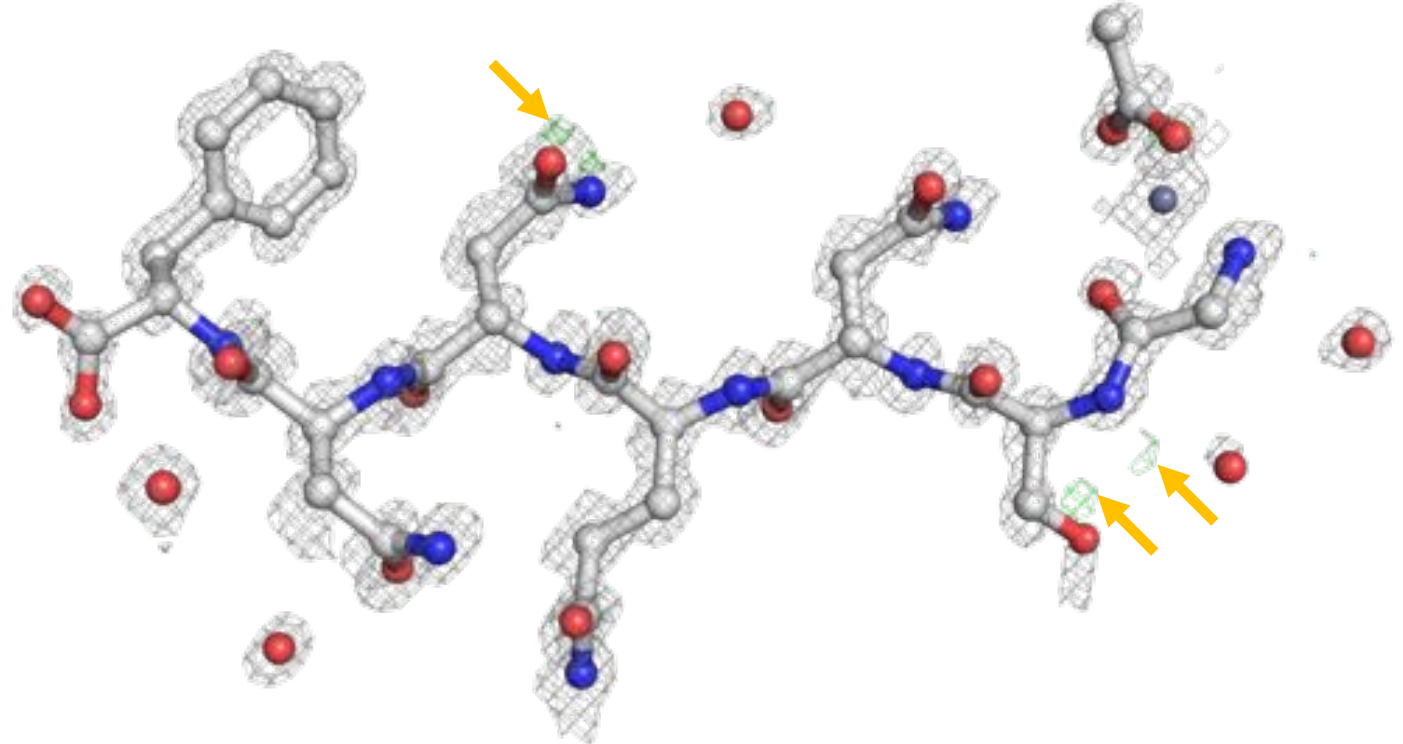

c.

Figure-1
B.

hPrP- $\beta 2 \alpha 2$ structure from thick crystals
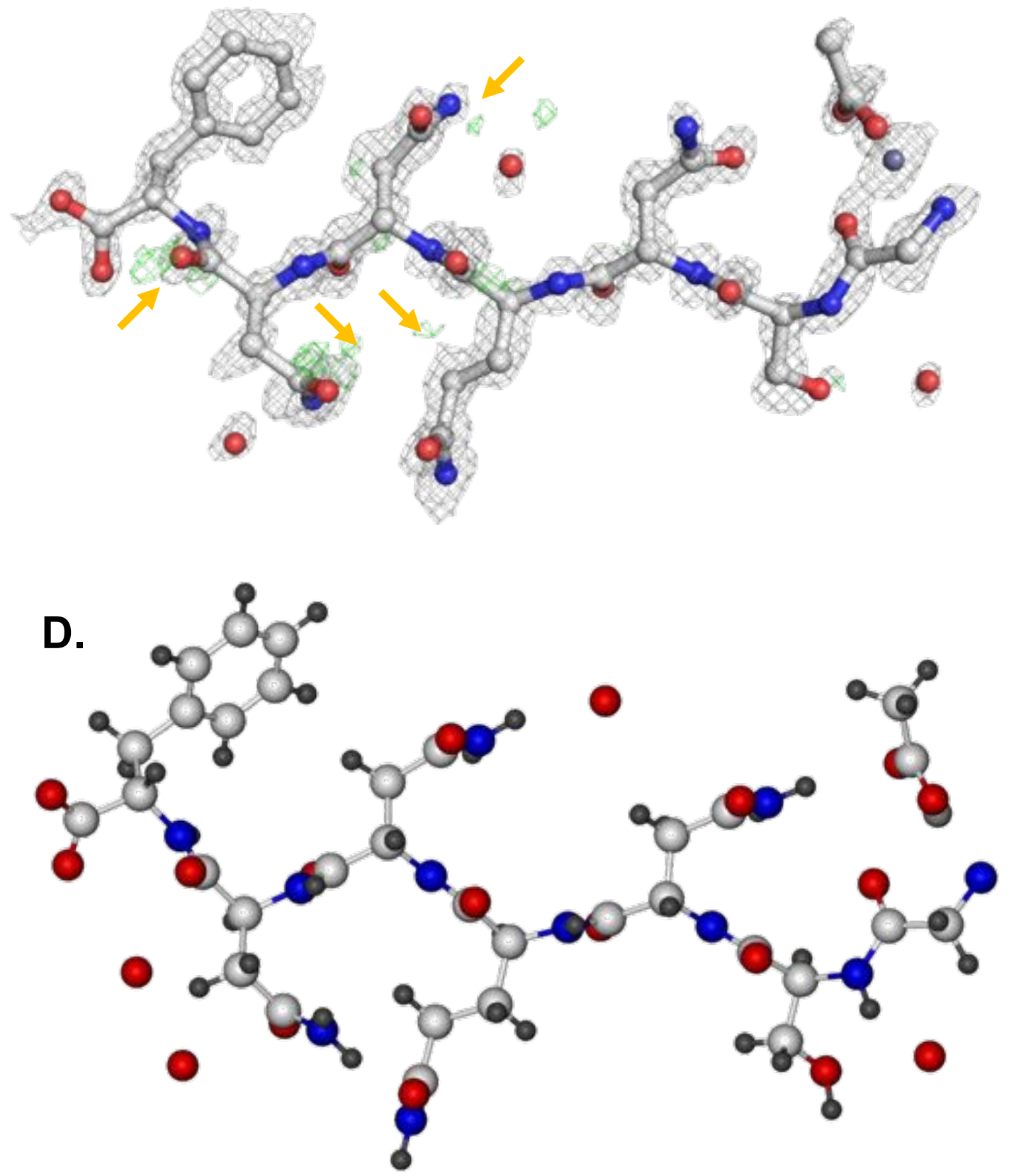
A.

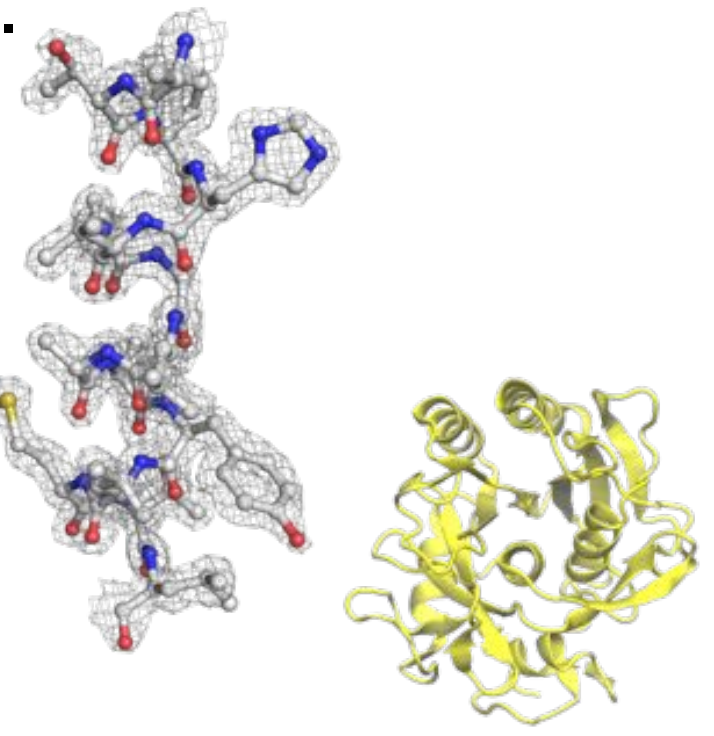

Four thin crystals
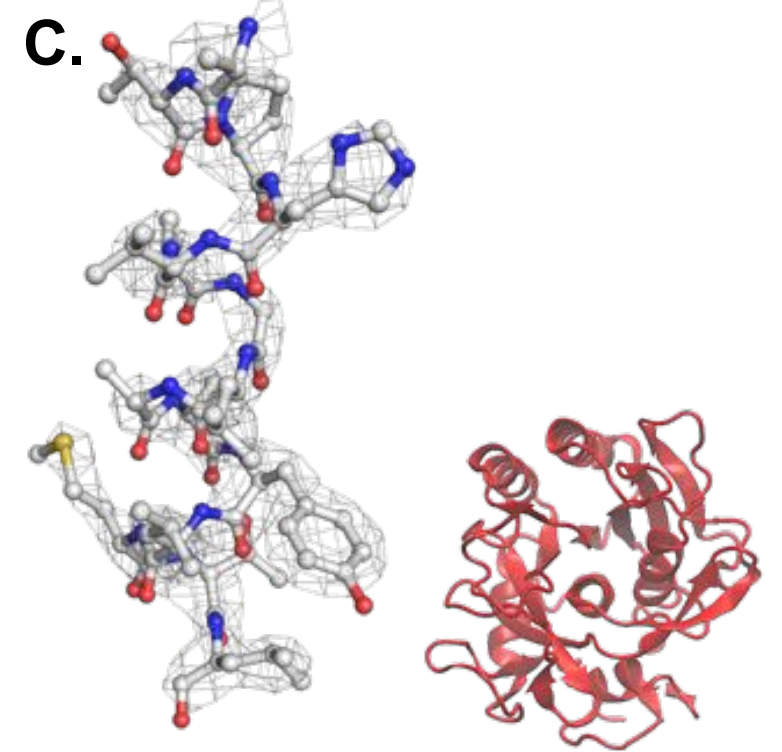

Single thick crystal
B.
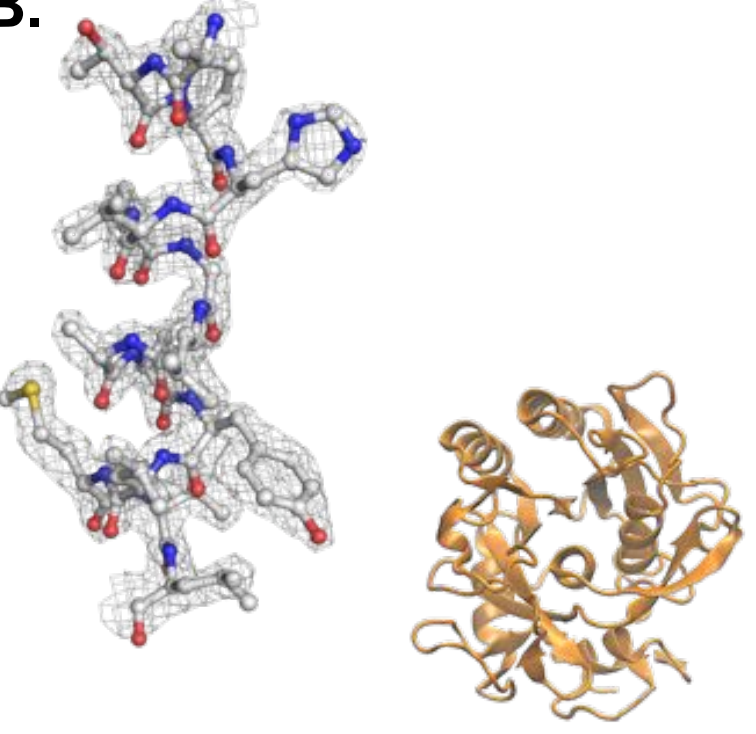

Single intermediate crystal

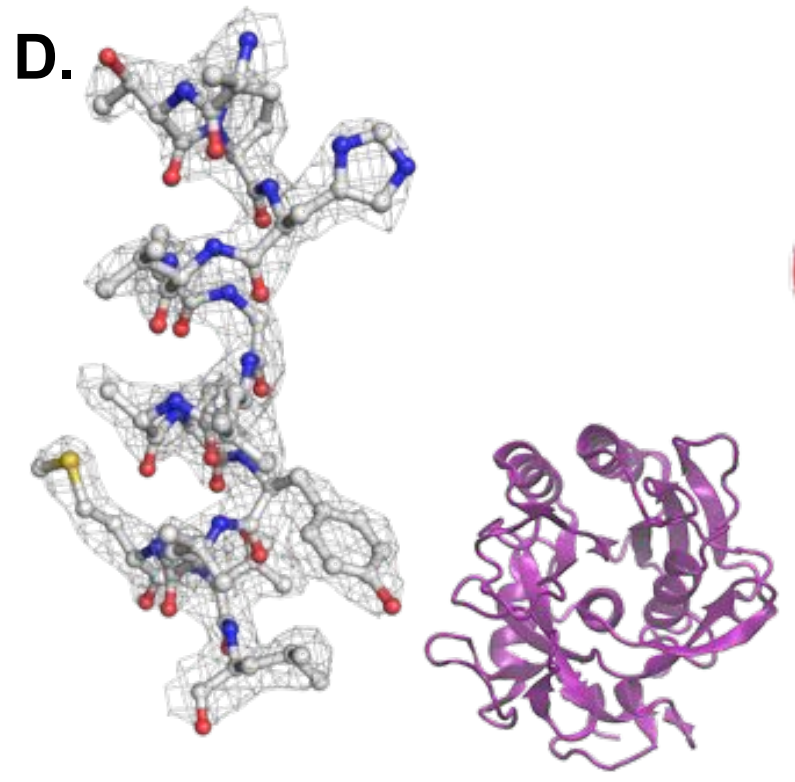

Four thick crystals
E.

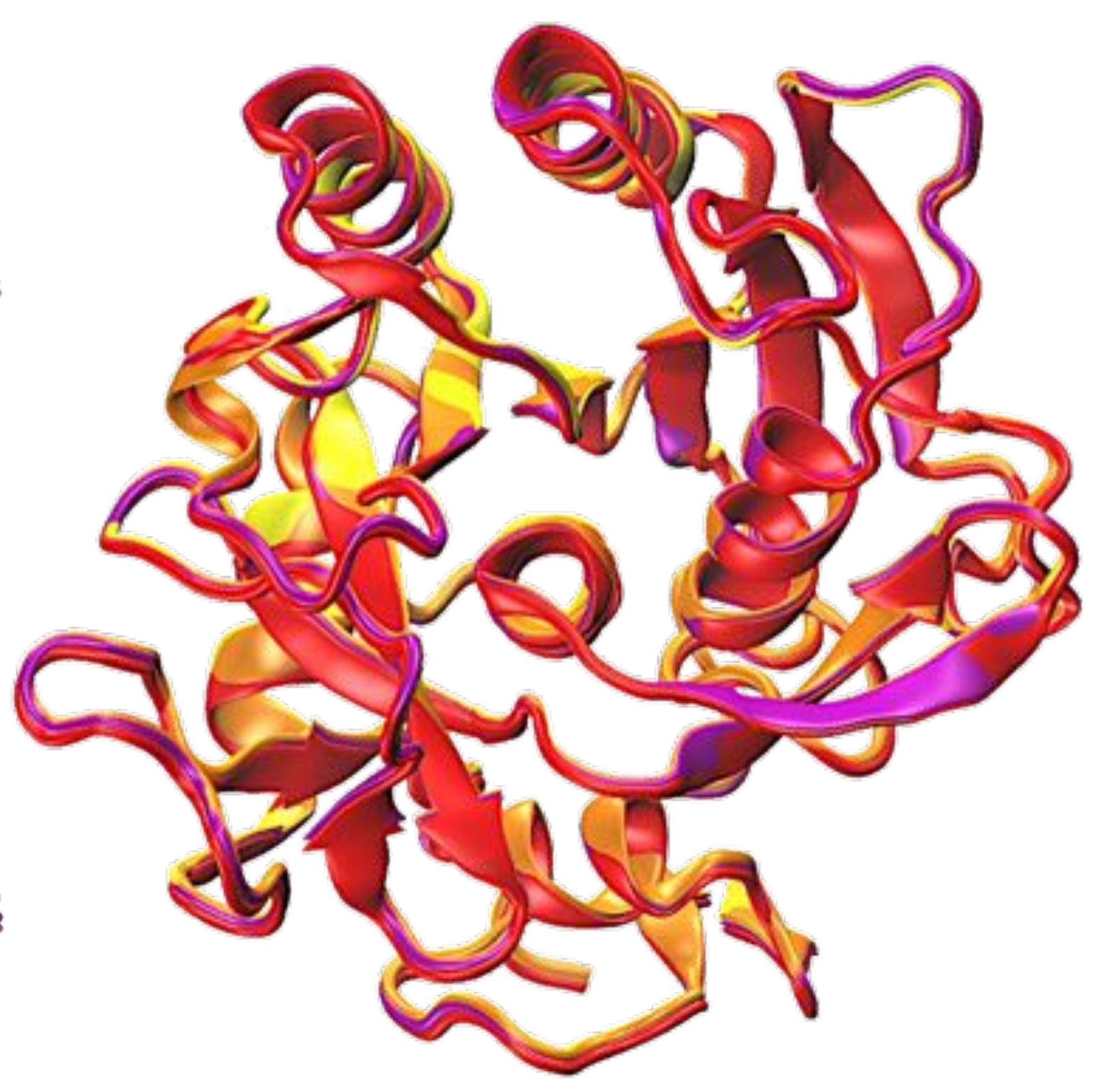

Figure-2 
Transmission vs. Crystal Thickness

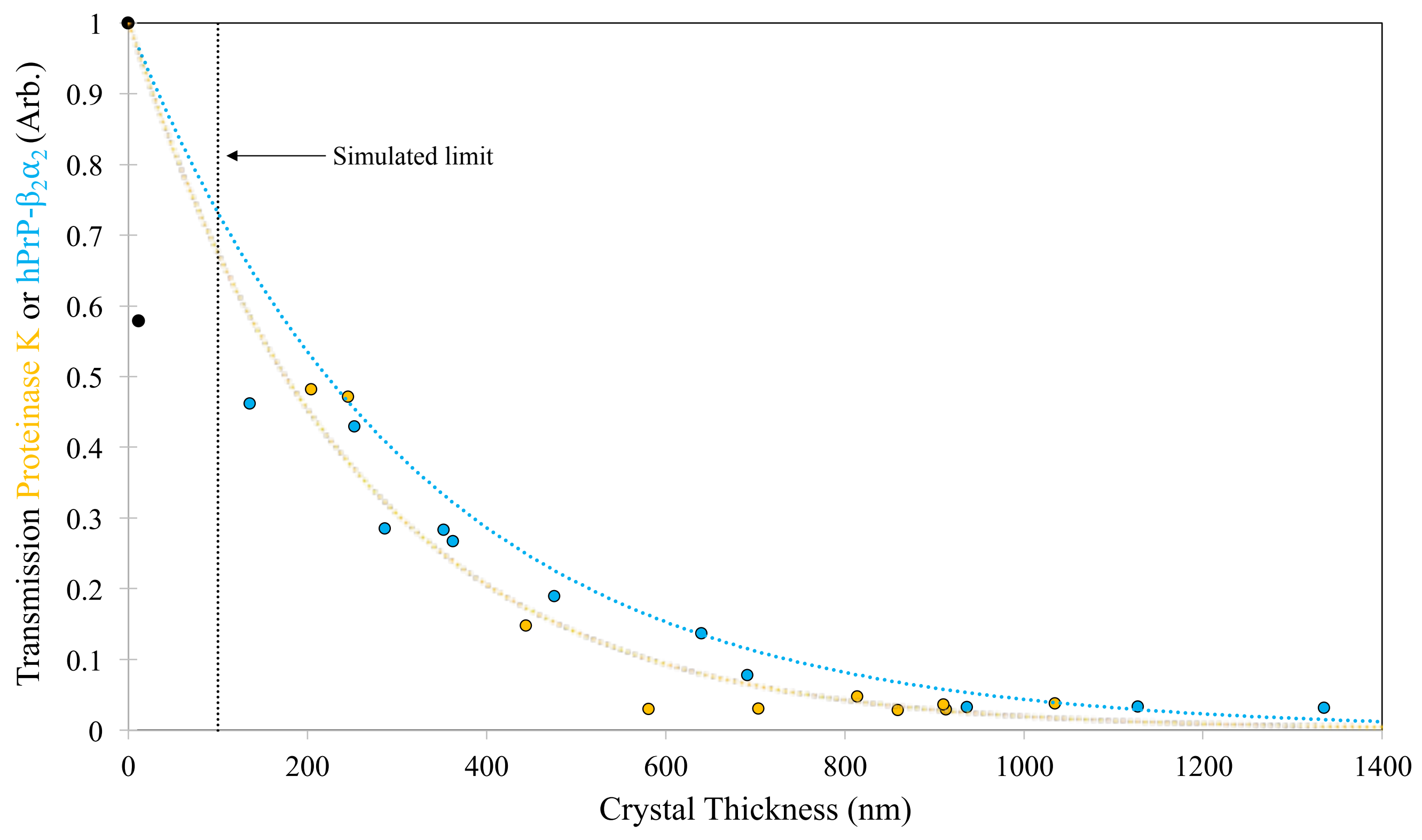

\section{Figure-4}


SI-Table-1: MicroED parameters for individual hPrP- $\beta 2 \alpha 2$ crystals

\begin{tabular}{|c|c|c|c|c|c|c|c|c|c|c|c|c|c|}
\hline $\begin{array}{c}\text { Mean } \\
\text { Thickness } \\
(\mathbf{n m}) \\
\end{array}$ & \# Observed & \# Unique & \# Possible & Completeness & $\mathbf{R}_{\text {observed }}$ & $\mathbf{R}_{\text {expected }}$ & \# Compared & $\mathbf{I} / \boldsymbol{\sigma I}$ & $\mathbf{R}_{\text {measured }}$ & $\mathrm{CC}_{1 / 2}$ & $\mathbf{C C}_{\text {model }}$ & $\mathbf{R}_{\text {difference }}$ & Crystal \# \\
\hline 91 & 1420 & 771 & 2625 & 0.29 & 0.08 & 0.09 & 1298.00 & 4.78 & 0.11 & 98.90 & 95.80 & 0.03 & 18 \\
\hline 106 & 921 & 518 & 2490 & 0.21 & 0.10 & 0.11 & 806.00 & 2.79 & 0.15 & 99.30 & 83.70 & 0.03 & 11 \\
\hline 145 & 1311 & 720 & 2391 & 0.30 & 0.10 & 0.11 & 1182.00 & 3.37 & 0.14 & 98.60 & 90.00 & 0.02 & 7 \\
\hline 150 & 1031 & 584 & 2622 & 0.22 & 0.11 & 0.12 & 894.00 & 3.14 & 0.15 & 98.40 & 88.10 & 0.03 & 20 \\
\hline 155 & 1266 & 676 & 2544 & 0.27 & 0.10 & 0.13 & 1180.00 & 3.46 & 0.14 & 98.30 & 89.00 & 0.02 & 12 \\
\hline 209 & 1390 & 747 & 2596 & 0.29 & 0.08 & 0.11 & 1286.00 & 3.82 & 0.11 & 99.20 & 95.20 & 0.01 & 5 \\
\hline 217 & 1313 & 700 & 2582 & 0.27 & 0.11 & 0.12 & 1226.00 & 2.19 & 0.16 & 98.20 & 90.70 & 0.03 & 13 \\
\hline 223 & 1396 & 767 & 2566 & 0.30 & 0.10 & 0.11 & 1258.00 & 3.67 & 0.14 & 97.50 & 93.20 & 0.03 & 8 \\
\hline 225 & 1280 & 690 & 2587 & 0.27 & 0.10 & 0.11 & 1180.00 & 4.07 & 0.14 & 98.00 & 71.80 & 0.03 & 3 \\
\hline 277 & 1415 & 775 & 2585 & 0.30 & 0.08 & 0.08 & 1280.00 & 5.74 & 0.11 & 98.30 & 97.10 & 0.03 & 21 \\
\hline 291 & 942 & 572 & 2543 & 0.23 & 0.06 & 0.07 & 740.00 & 3.59 & 0.09 & 99.60 & 80.80 & 0.02 & 14 \\
\hline 360 & 1471 & 790 & 2624 & 0.30 & 0.08 & 0.09 & 1362.00 & 4.97 & 0.11 & 98.10 & 96.00 & 0.03 & 9 \\
\hline 372 & 1419 & 769 & 2526 & 0.30 & 0.10 & 0.12 & 1300.00 & 3.83 & 0.14 & 98.50 & 93.00 & 0.02 & 17 \\
\hline 437 & 1351 & 725 & 2526 & 0.29 & 0.09 & 0.10 & 1252.00 & 4.04 & 0.13 & 98.00 & 92.30 & 0.03 & 10 \\
\hline 481 & 1280 & 689 & 2665 & 0.26 & 0.06 & 0.08 & 1182.00 & 5.54 & 0.09 & 99.40 & 85.60 & 0.01 & 1 \\
\hline 578 & 1505 & 816 & 2644 & 0.31 & 0.09 & 0.11 & 1378.00 & 4.05 & 0.13 & 98.30 & 94.60 & 0.02 & 16 \\
\hline 664 & 1504 & 796 & 2554 & 0.31 & 0.07 & 0.08 & 1416.00 & 5.34 & 0.11 & 98.80 & 93.70 & 0.02 & 2 \\
\hline 1012 & 1157 & 597 & 2593 & 0.23 & 0.10 & 0.11 & 1060.00 & 3.77 & 0.14 & 96.80 & 91.50 & 0.03 & 15 \\
\hline 1107 & 686 & 378 & 2180 & 0.17 & 0.13 & 0.13 & 616.00 & 3.99 & 0.19 & 93.20 & 77.70 & 0.06 & 19 \\
\hline
\end{tabular}

\section{SI-Table-1}


A.
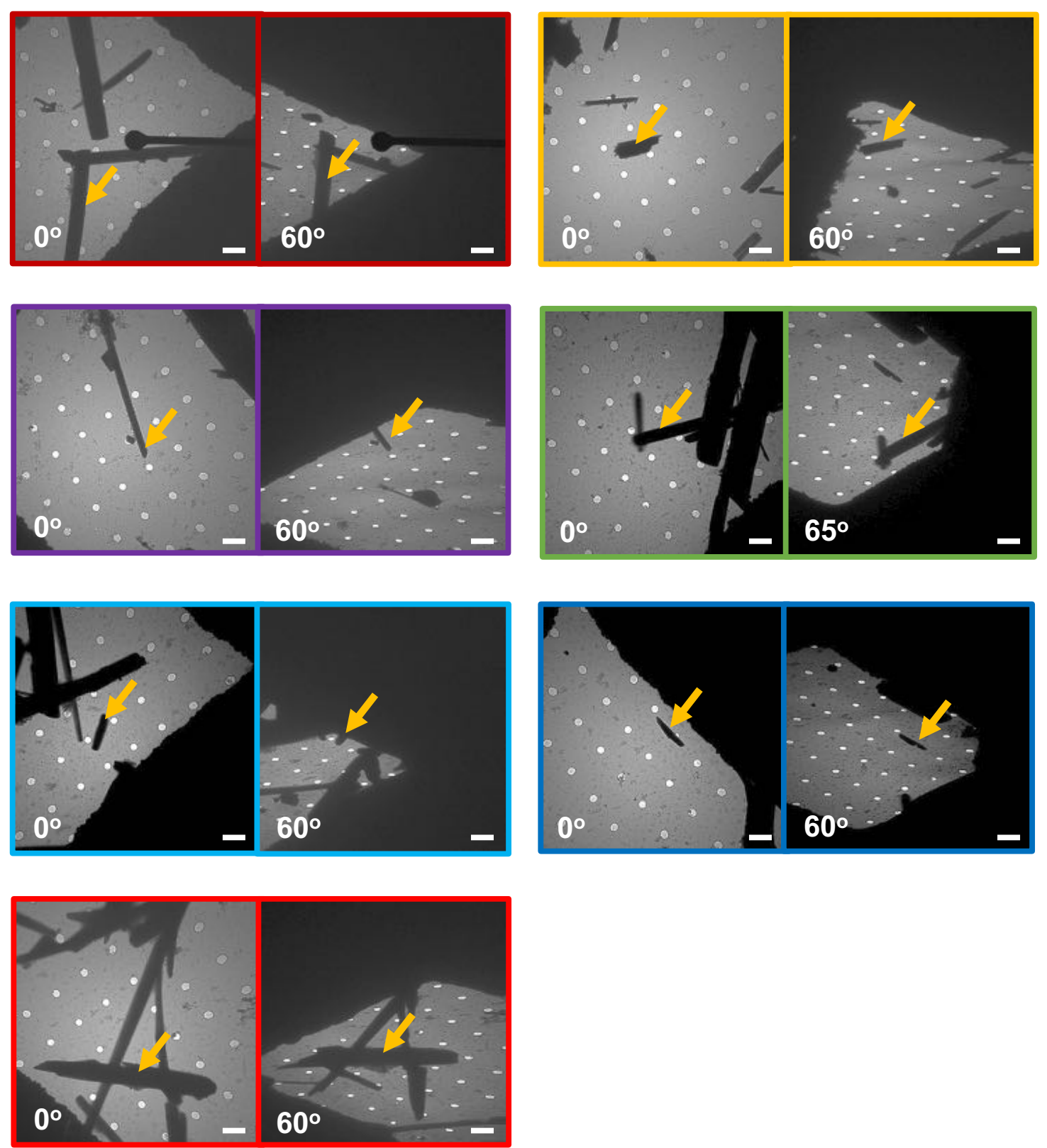

\section{SI-Figure-1}

B.

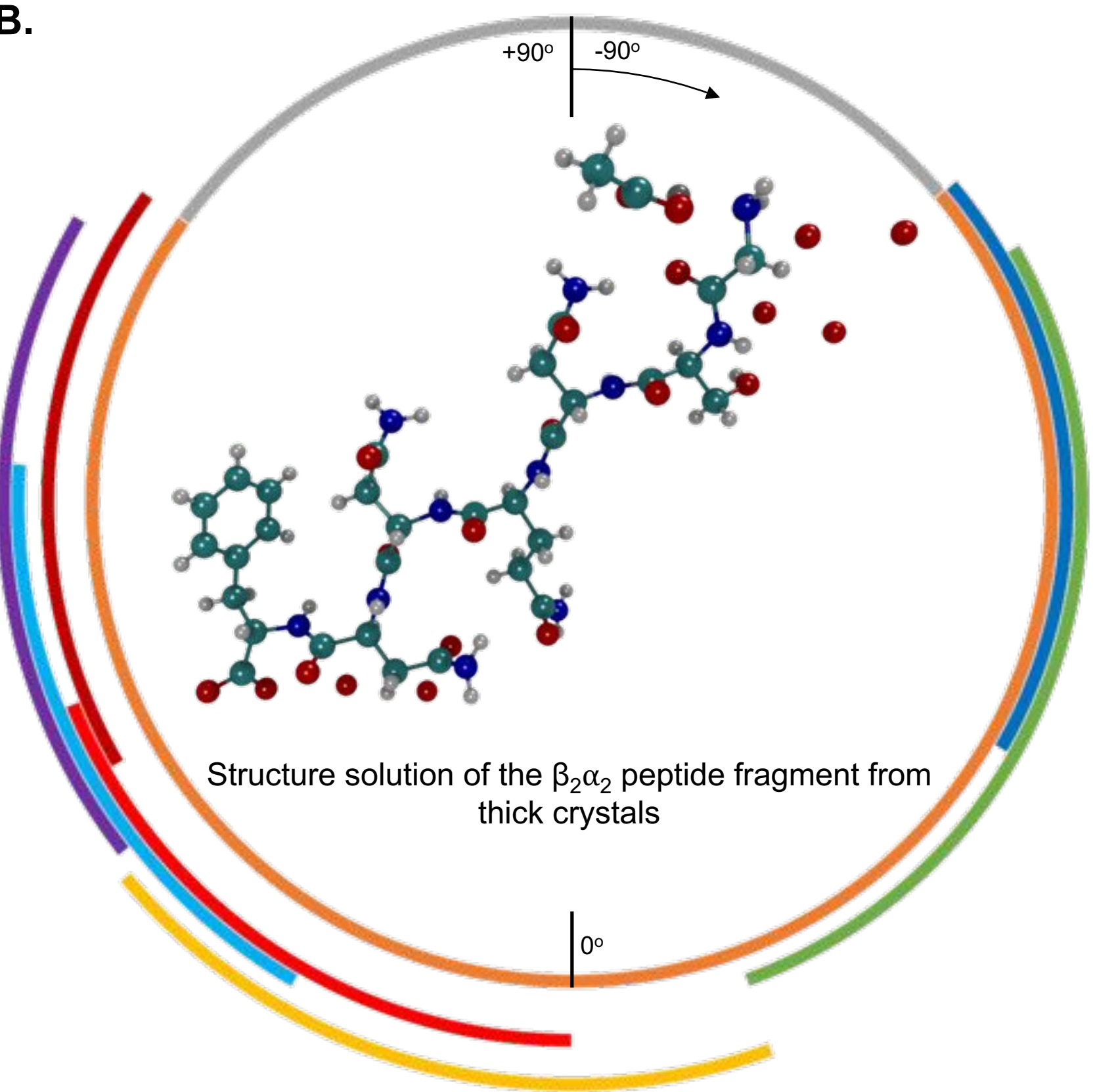


A.

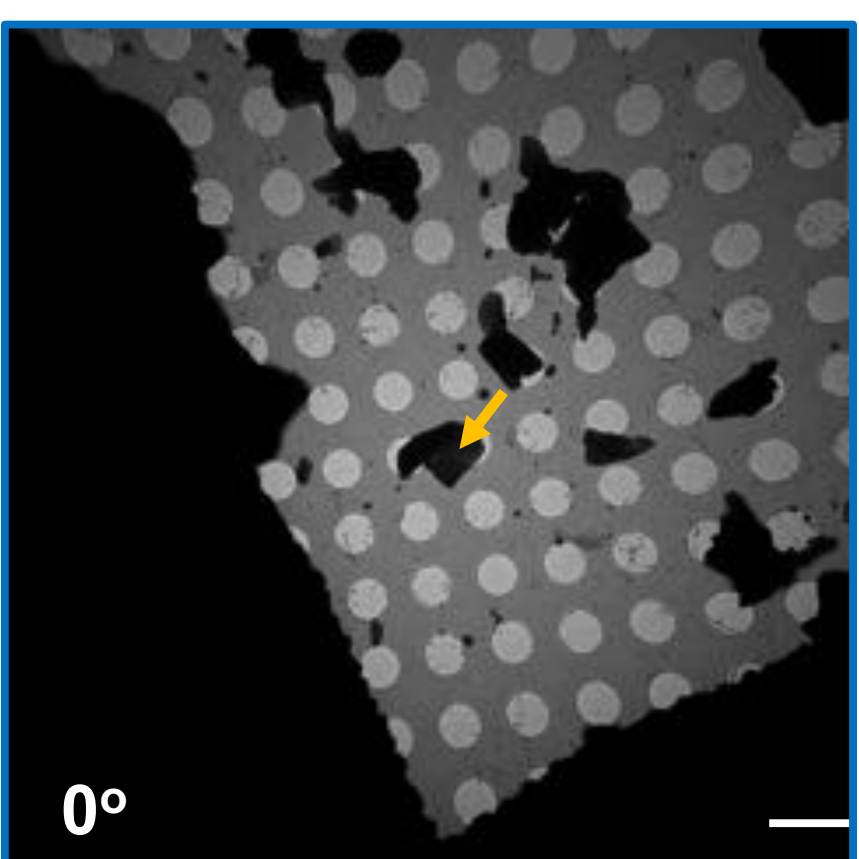

$60^{\circ}$

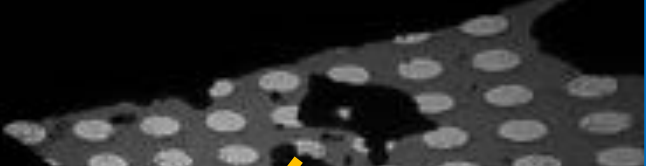

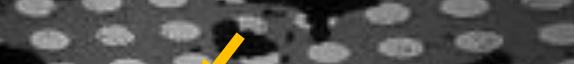

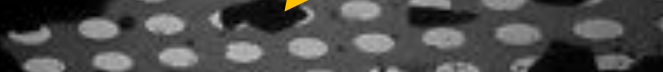
$-2038294$ 80805002 800
B.

$+30^{\circ}$

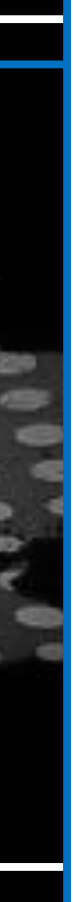

\section{SI-Figure-2}


A.
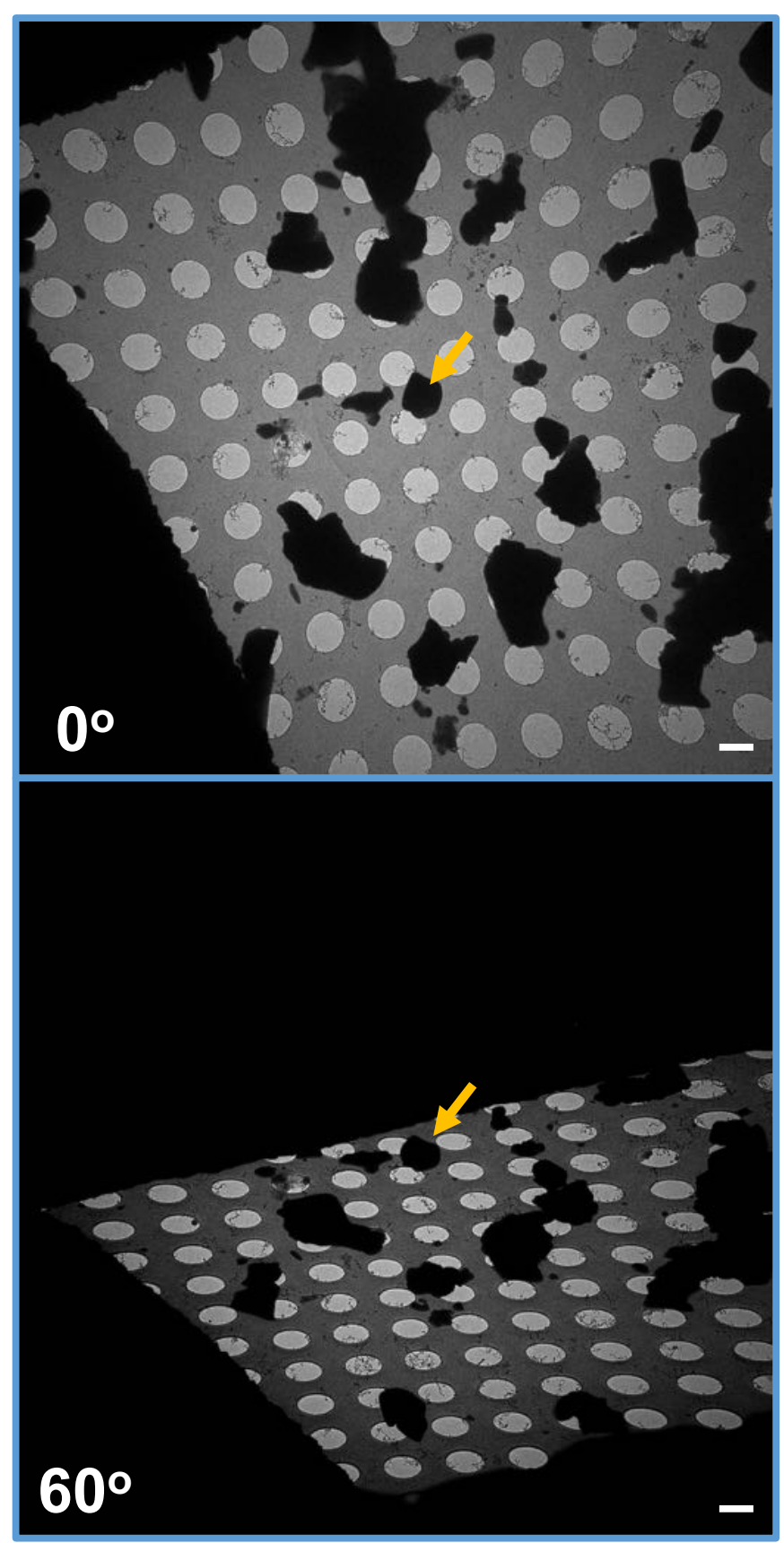

B.

$+30^{\circ}$

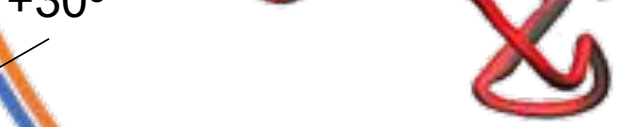

Structure of Proteinase $\mathrm{K}$ from a single thick crystal 
A.
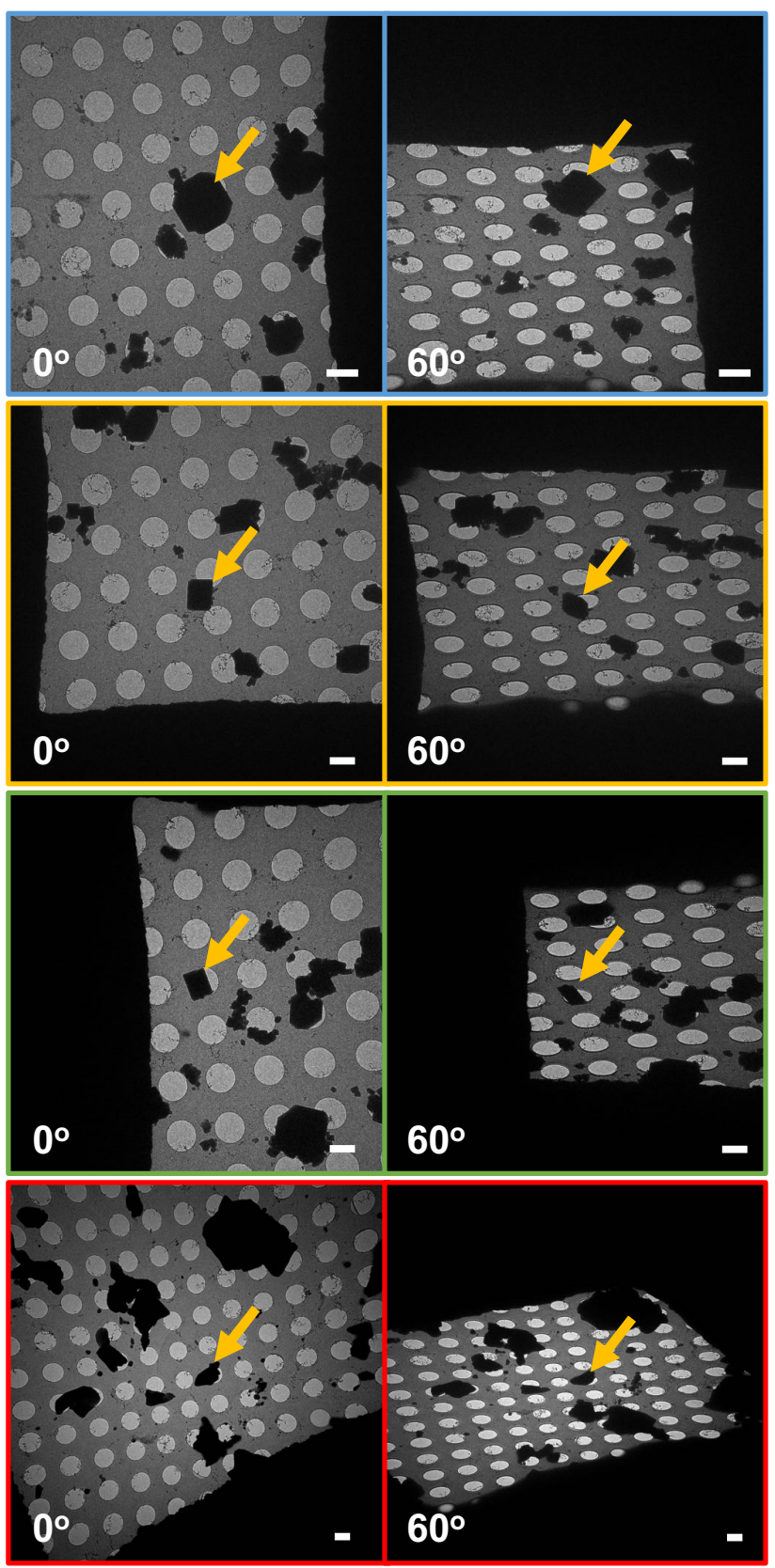

B.

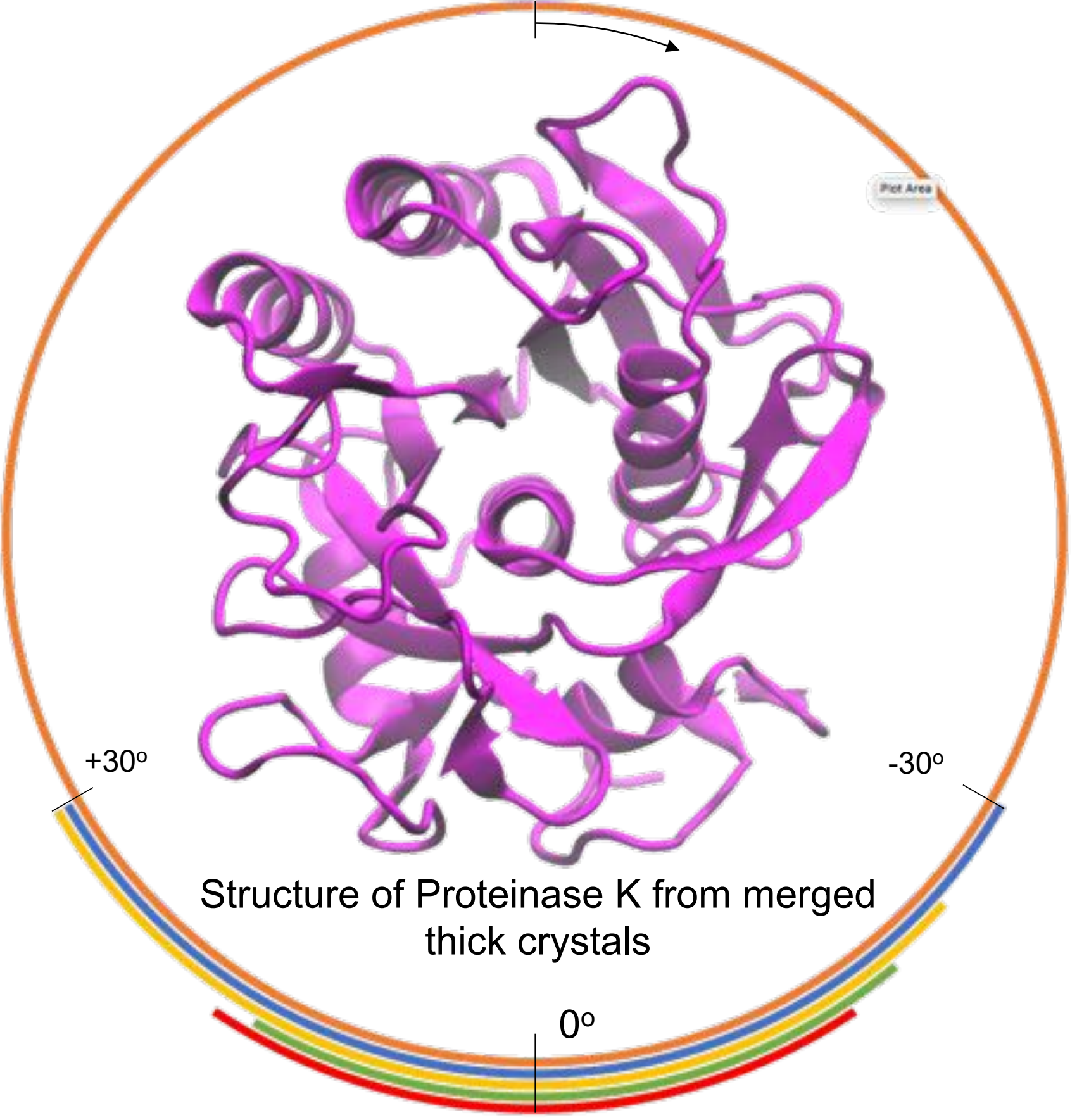


A.

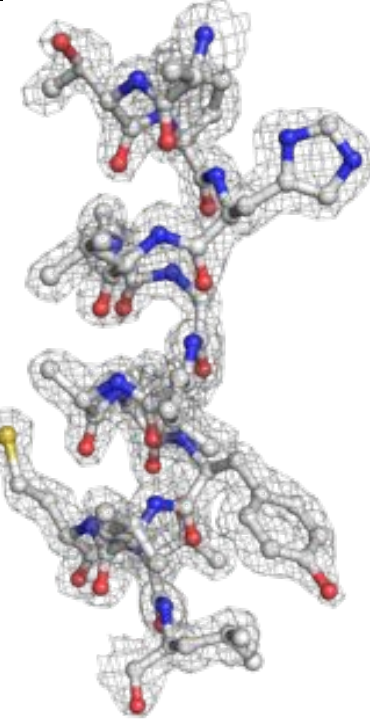

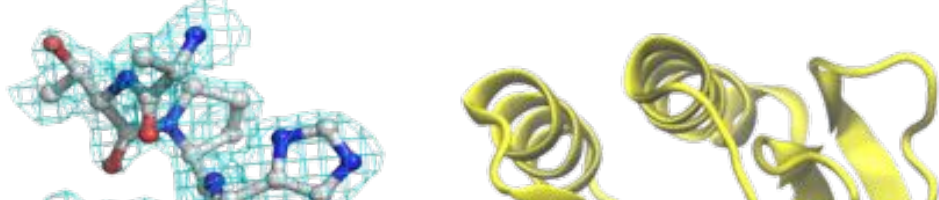

$\frac{2^{2}}{y^{2}}$

cepose

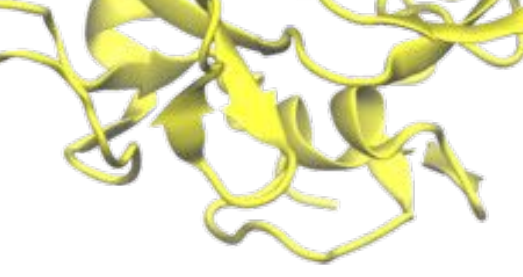

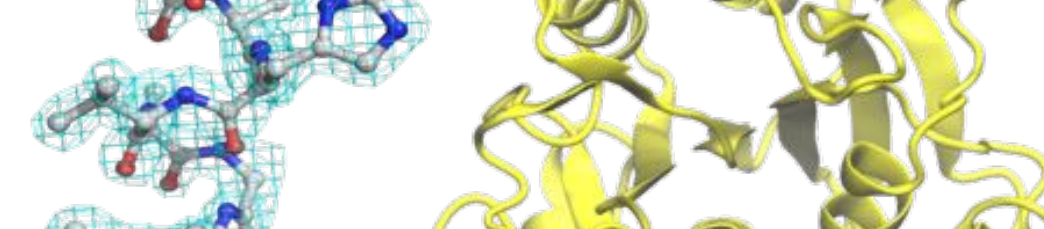

B.

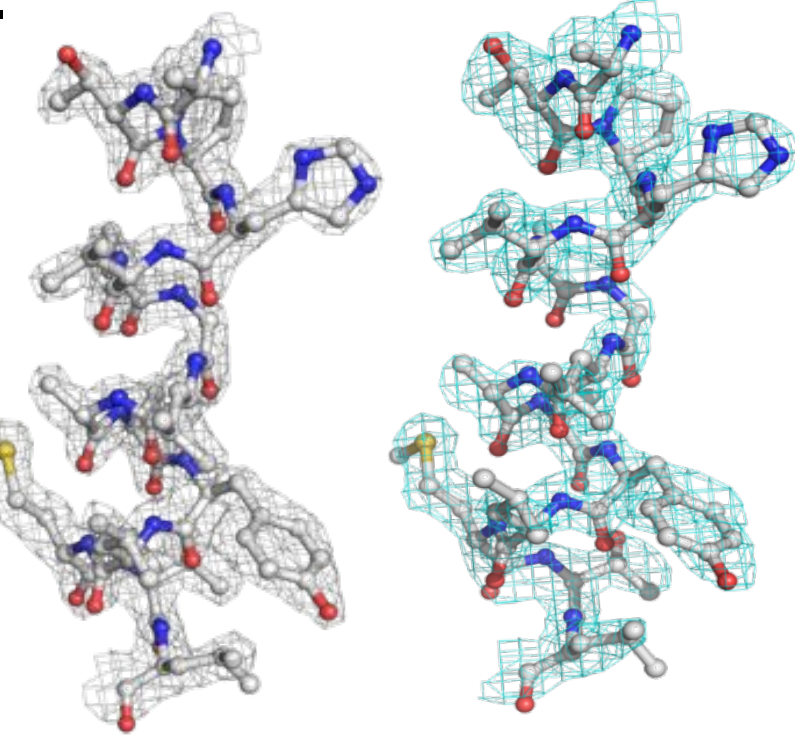

D.

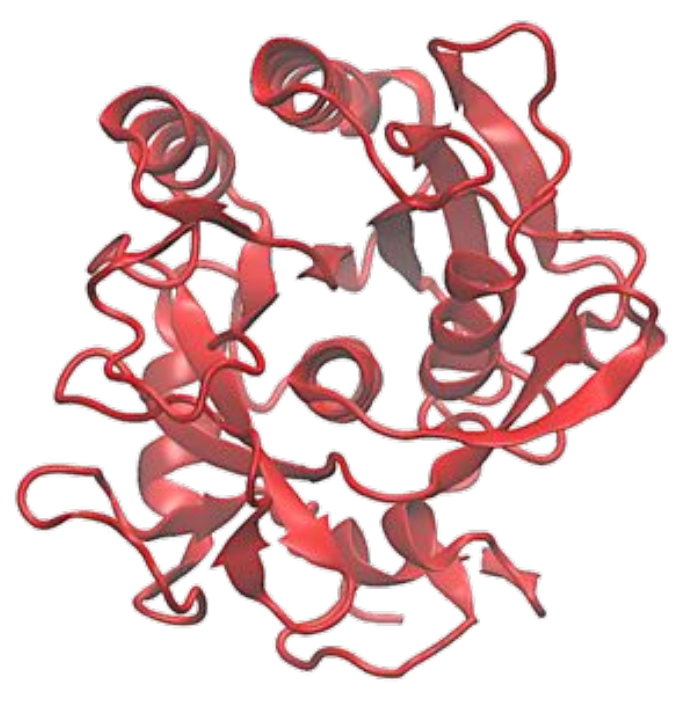

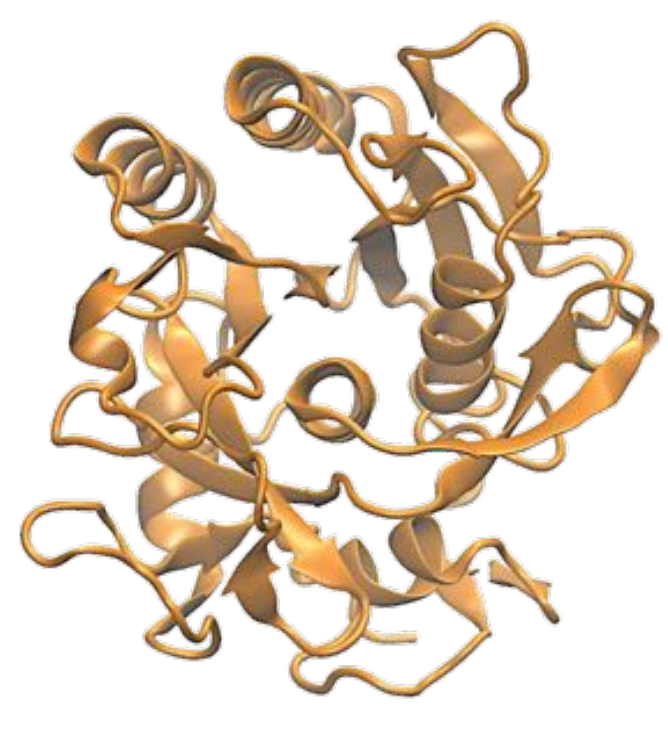

c.
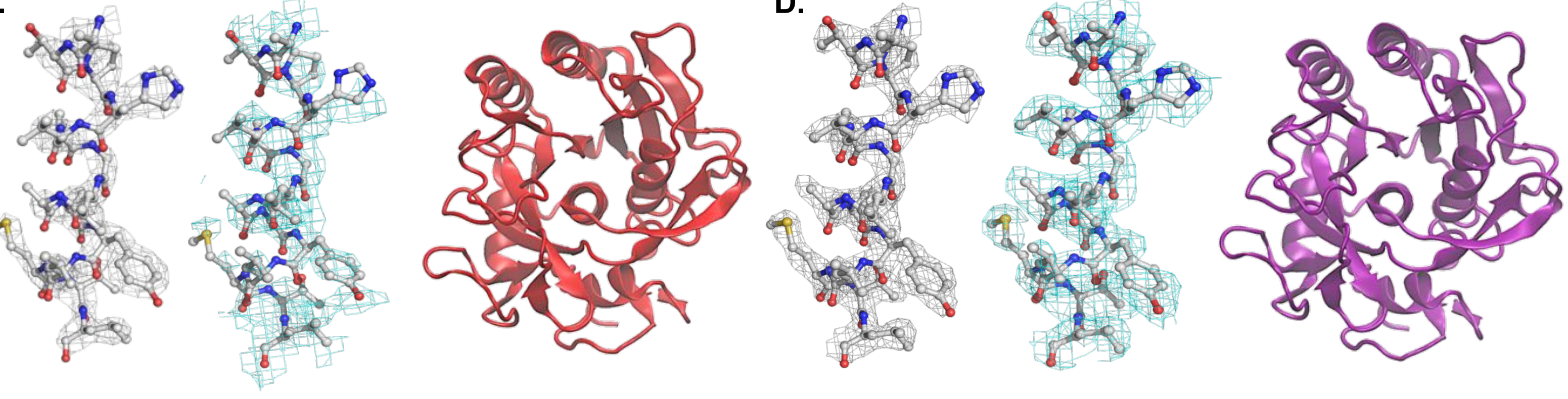

\section{SI-Figure-5}


A.

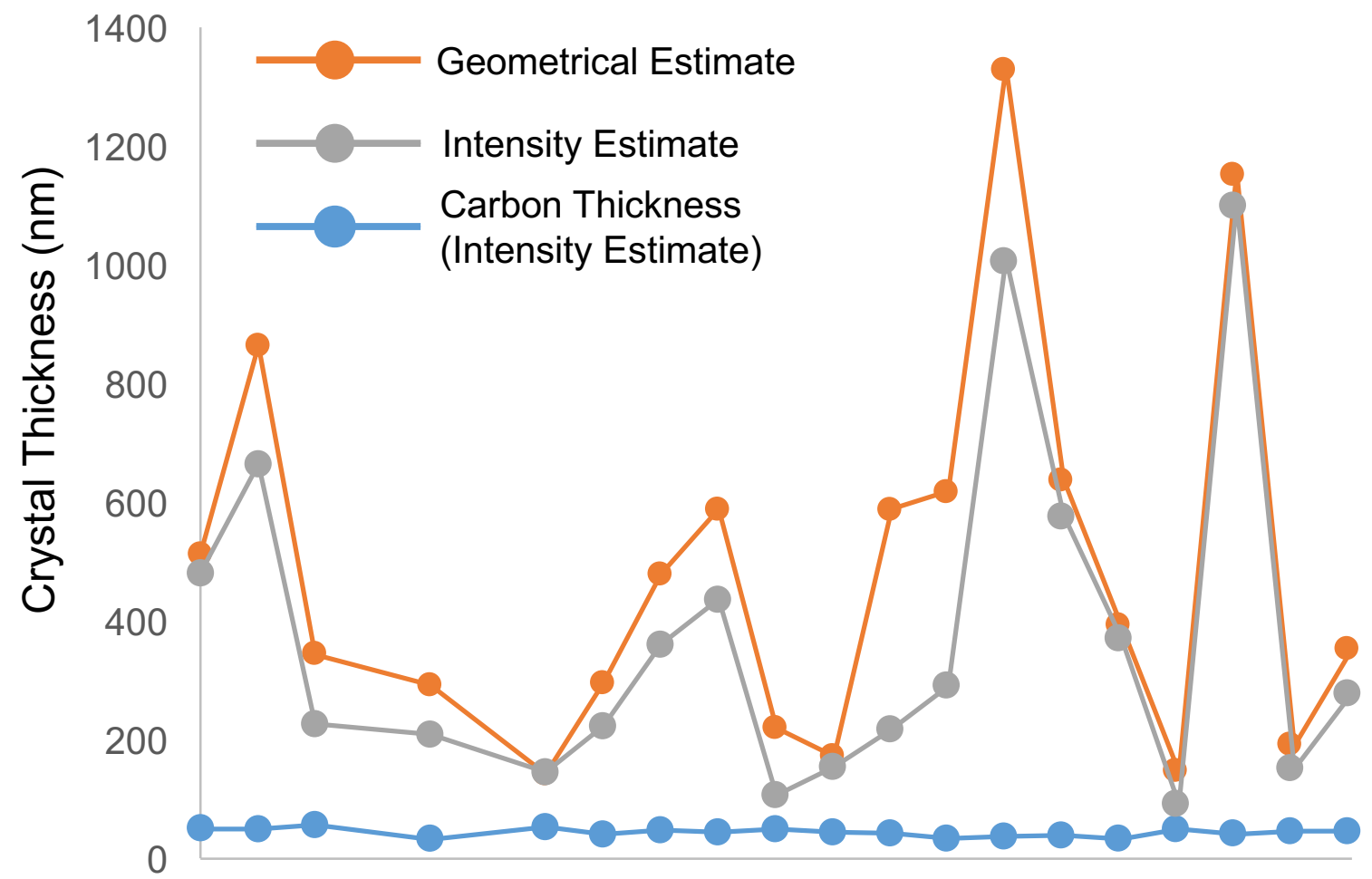

$\begin{array}{lllllllllllllllllllll}1 & 2 & 3 & 4 & 5 & 6 & 7 & 8 & 9 & 10 & 11 & 12 & 13 & 14 & 15 & 16 & 17 & 18 & 19 & 20 & 21\end{array}$ Crystal \#
B.

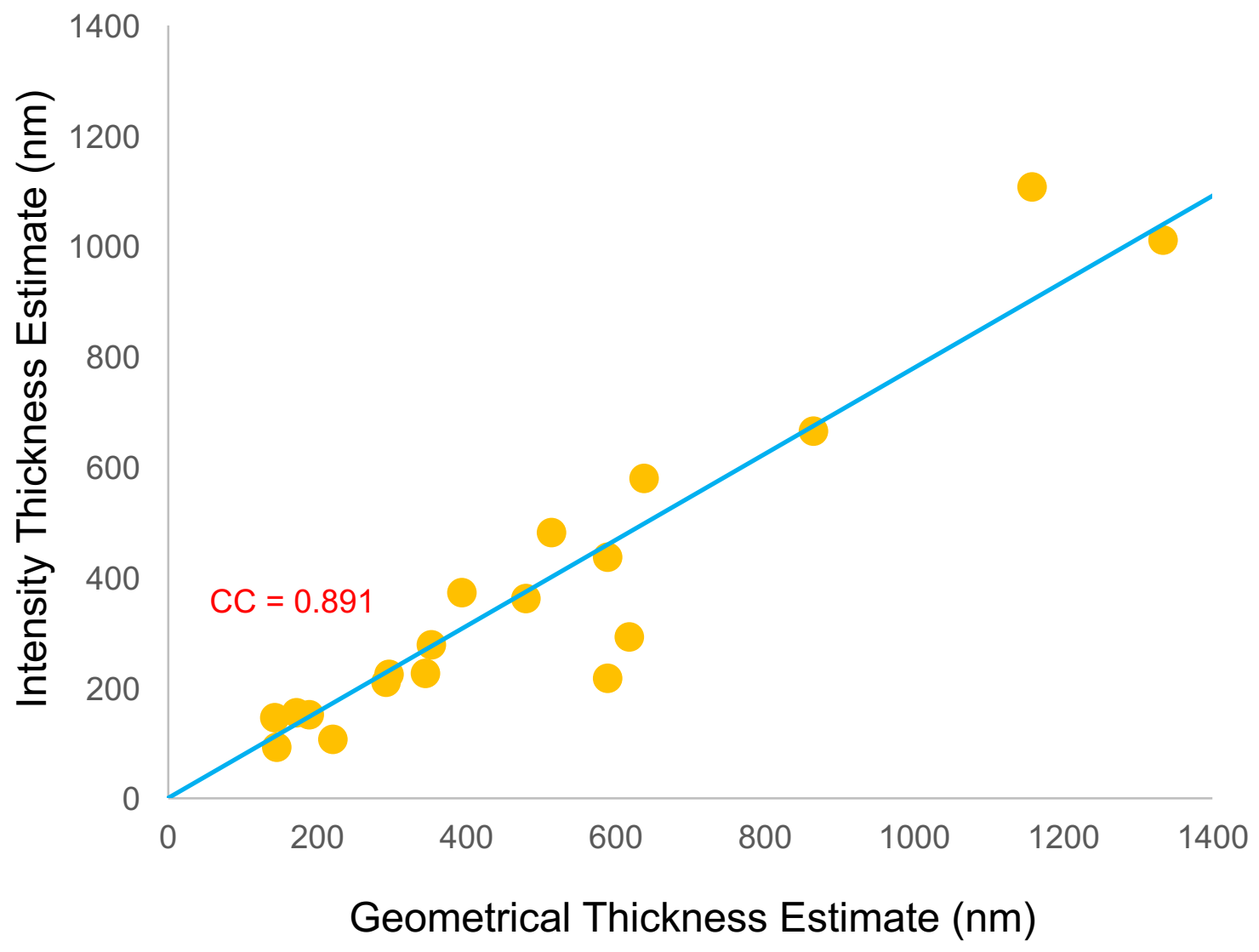


SI-Document-2: Peptide tilt angle images (diffraction data crystals) - PDF

SI-Document-3: EELS Data (images and fits) - PDF 
Crystals of hPrP - $\beta_{2} \alpha_{2}$ used to generate statistics in Figure-3 and SI-Table-1
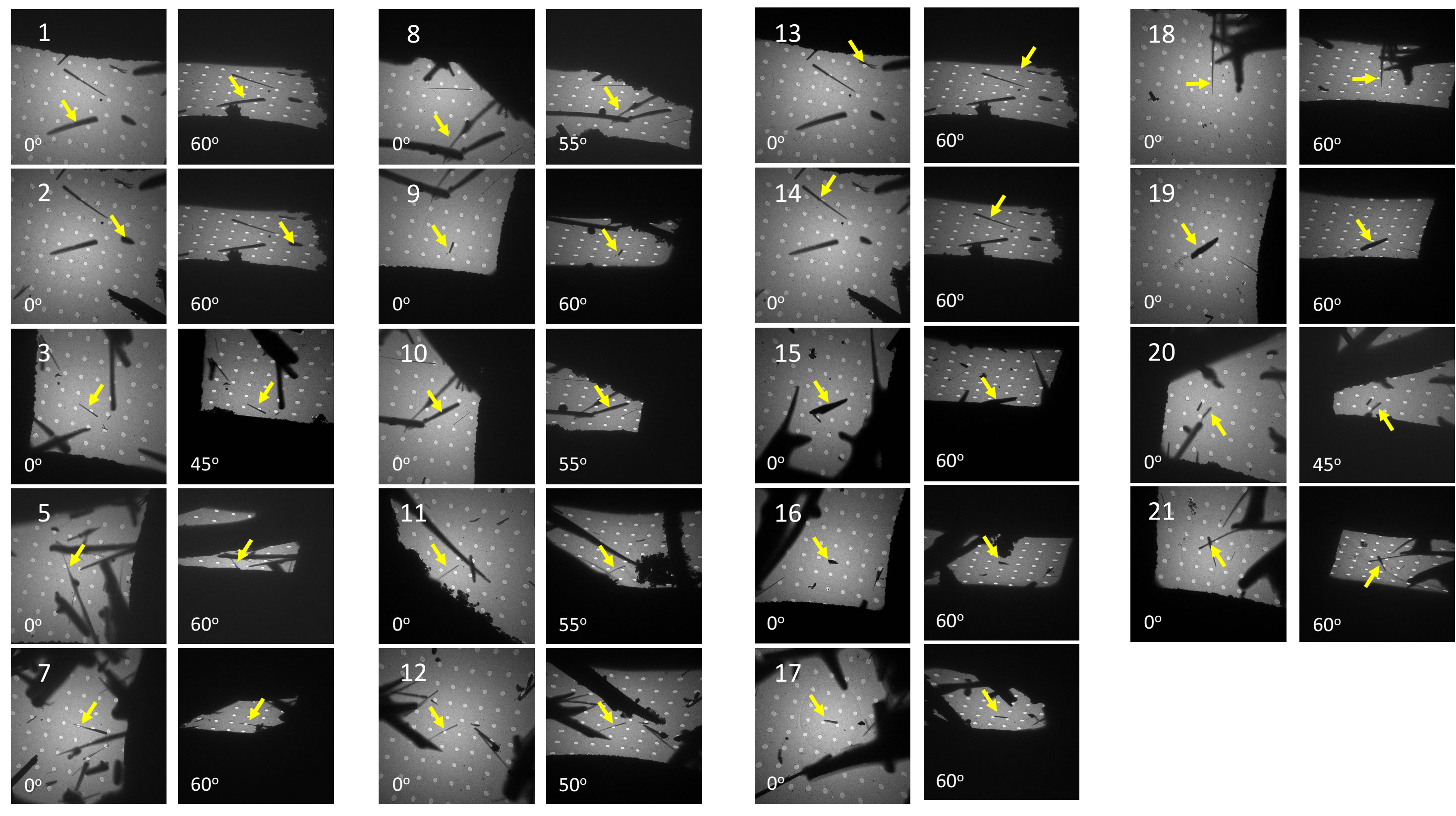
hPrP - $B_{2} \alpha_{2}$ Crystals used in EELS Experiments
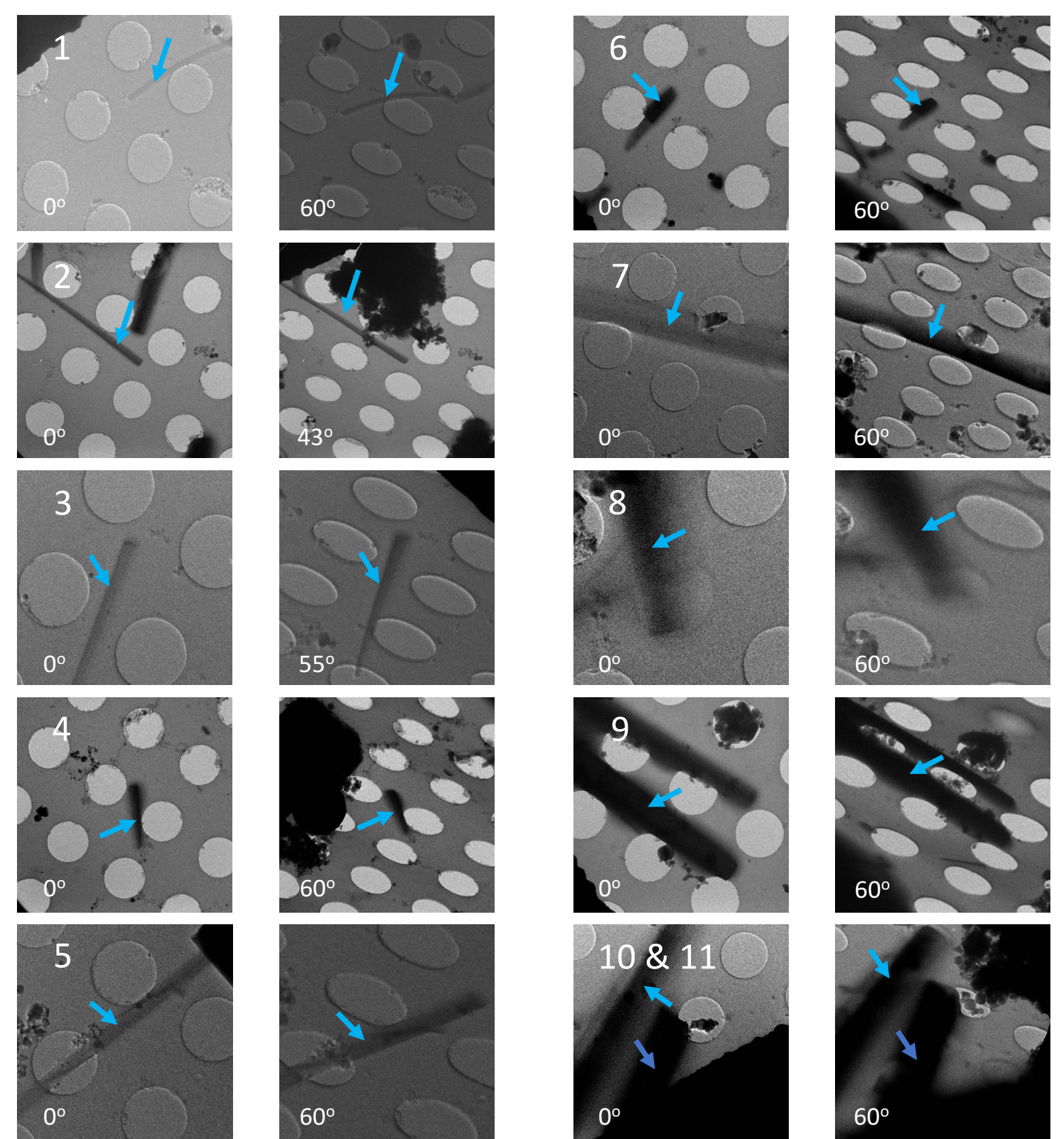
Proteinase $\mathrm{K}$ Crystals in EELS Experiments

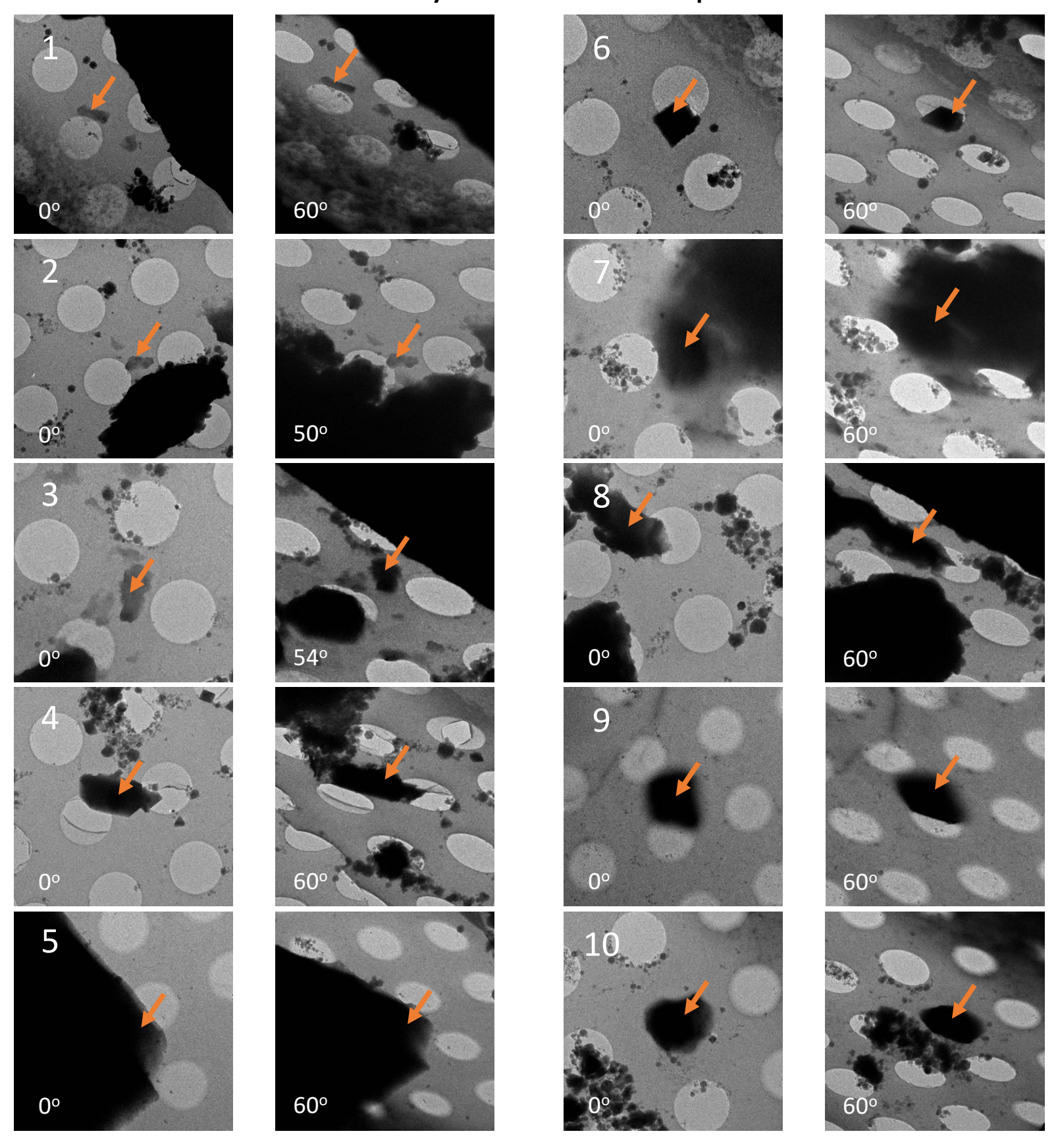


EELS Spectra from $\beta_{2} \alpha_{2}$ crystals at $300 \mathrm{kV}$

\begin{tabular}{|c|c|c|c|}
\hline $\begin{array}{l}\text { Vacuum } \\
\\
0 \mathrm{~nm} \\
0.9 \mathrm{~s}\end{array}$ & $\begin{array}{l}1 \\
\\
\\
134 \mathrm{~nm} \\
1 \mathrm{~s}\end{array}$ & $\begin{array}{l}360 \mathrm{~nm} \\
1 \mathrm{~s}\end{array}$ & $\begin{array}{l}9 \\
\\
\\
931 \mathrm{~nm} \\
5 s\end{array}$ \\
\hline $\begin{array}{l}\text { Carbon Foil } \\
\\
45 \mathrm{~nm} \\
1 \mathrm{~s}\end{array}$ & $\begin{array}{l}2 \\
\\
\\
249 \mathrm{~nm} \\
1 \mathrm{~s}\end{array}$ & $\begin{array}{l}471 \mathrm{~nm} \\
1 \mathrm{~s}\end{array}$ & $\begin{array}{l}10 \\
\\
\\
1120 \mathrm{~nm} \\
5 s\end{array}$ \\
\hline & $\begin{array}{l}3 \\
\\
\\
\\
283 \mathrm{~nm} \\
1 \mathrm{~s}\end{array}$ & $\begin{array}{l}7 \\
\\
\\
635 \mathrm{~nm} \\
5 s\end{array}$ & $\begin{array}{l}11 \\
\\
1330 \mathrm{~nm} \\
1 \mathrm{~s}\end{array}$ \\
\hline & $\begin{array}{l}348 \mathrm{~nm} \\
1 \mathrm{~s}\end{array}$ & $\begin{array}{l}8 \\
\\
\\
686 \mathrm{~nm} \\
1 \mathrm{~s}\end{array}$ & \\
\hline
\end{tabular}


EELS Spectra from Proteinase K Crystals at 300kV

\begin{tabular}{|c|c|c|c|}
\hline $\begin{array}{l}\text { Vacuum } \\
\\
0 \mathrm{~nm} \\
0.9 \mathrm{~s}\end{array}$ & $\begin{array}{l}1 \\
\\
\\
259 \mathrm{~nm} \\
1 \mathrm{~s} \\
\end{array}$ & $\begin{array}{l}5 \\
\\
\\
\\
896 \mathrm{~nm} \\
1 \mathrm{~s} \\
\end{array}$ & $\begin{array}{l}9 \\
\\
\\
1163 \mathrm{~nm} \\
5 s \\
\end{array}$ \\
\hline $\begin{array}{l}\text { Carbon Foil } \\
\\
45 \mathrm{~nm} \\
1 \mathrm{~s}\end{array}$ & $\begin{array}{l}2 \\
\\
\\
310 \mathrm{~nm} \\
1 \mathrm{~s}\end{array}$ & $\begin{array}{l}6 \\
\\
\\
\\
1037 \mathrm{~nm} \\
5 s \\
\end{array}$ & $\begin{array}{l}10 \\
\\
\\
1318 \mathrm{~nm} \\
1 \mathrm{~s} *\end{array}$ \\
\hline & $\begin{array}{c}3 \\
\\
\\
564 \mathrm{~nm} \\
1 \mathrm{~s} \\
4\end{array}$ & $\begin{array}{l}7 \\
\\
\\
1095 \mathrm{~nm} \\
1 \mathrm{~s} \\
8 \\
\\
1160 \mathrm{~nm} \\
5 \mathrm{~s}\end{array}$ & \\
\hline
\end{tabular}


Line Scans of EELS Spectra for hPrP $-\beta_{2} \alpha_{2}$ at $300 \mathrm{kV}$
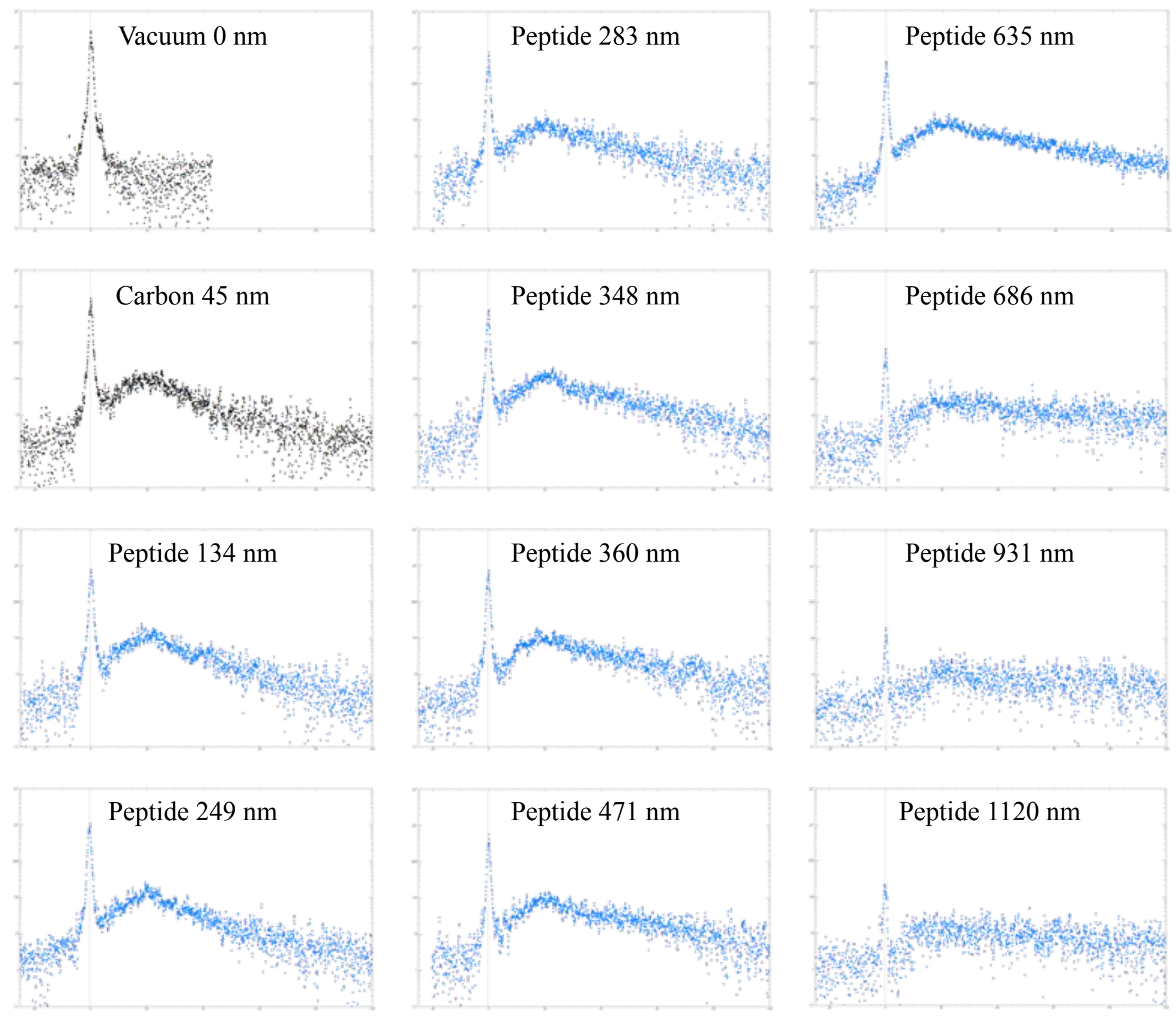


\section{Line Scans of EELS Spectra for Proteinase K Crystals}
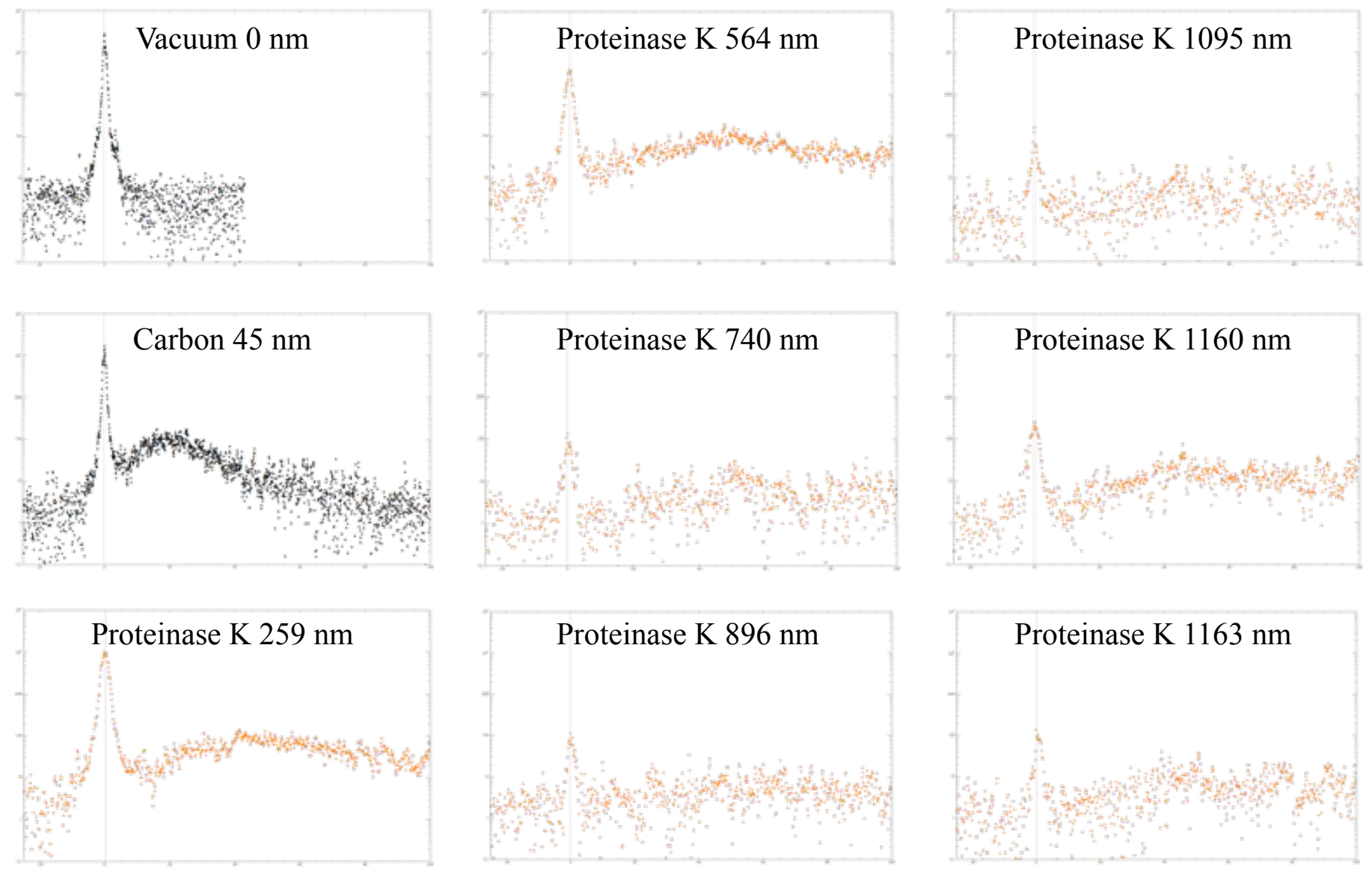

Proteinase K 310 nm

Proteinase K 1037 nm

Proteinase K 1318 nm
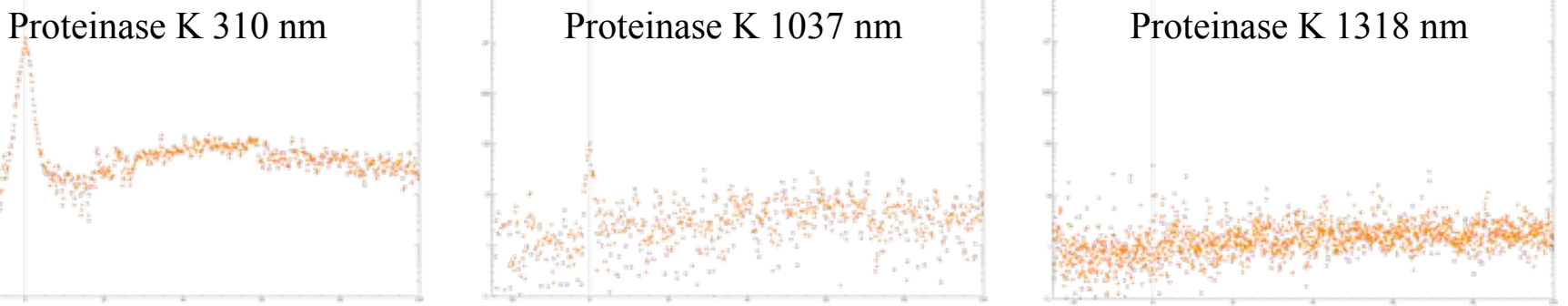